Grant Number: NAG 1-01037

INTERNATIONAL JOURNAL OF

COMPUTATIONAL

ENGINEERING

SCIENCE (IJCES)

Volume 4 • Number 1 - March 2003

Fully Nonlinear Modeling and Analysis of Precision Membranes

P. F. Pai and L. G. Young 


\title{
FULLY NONLINEAR MODELING AND ANALYSIS OF PRECISION MEMBRANES
}

\author{
P. FRANK PAI and LEYLAND G. YOUNG \\ Department of Mechanical and Aerospace Engineering, \\ University of Missouri-Columbia, \\ Columbia, MO 65211, USA \\ paip@missouri.edu
}

Received 26 August 2002

Accepted 4 November 2002

\begin{abstract}
High precision membranes are used in many current space applications. This paper presents a fully nonlinear membrane theory with forward and inverse analyses of high precision membrane structures. The fully nonlinear membrane theory is derived from Jaumann strains and stresses, exact coordinate transformations, the concept of local relative displacements, and orthogonal virtual rotations. In this theory, energy and Newtonian formulations are fully correlated, and every structural term can be interpreted in terms of vectors. Fully nonlinear ordinary differential equations (ODEs) governing the large static deformations of known axisymmetric membranes under known axisymmetric loading (i.e., forward problems) are presented as first-order ODEs, and a method for obtaining numerically exact solutions using the multiple shooting procedure is shown. A method for obtaining the undeformed geometry of any axisymmetric membrane with a known inflated geometry and a known internal pressure (i.e., inverse problems) is also derived. Numerical results from forward analysis are verified using results in the literature, and results from inverse analysis are verified using known exact solutions and solutions from the forward analysis. Results show that the membrane theory and the proposed numerical methods for solving nonlinear forward and inverse membrane problems are accurate.
\end{abstract}

Keywonds: Nonlinear membrane theory; numerically exact analysis.

\section{Introduction}

As the cargo space of a launch vehicle is limited, large spacecraft structures must be designed to be stowed during launch and deployed once on orbit. Moreover, as the launch expenditure of a NASA space mission always constitutes a significant fraction of the total cost, inexpensive launch vehicles with small payload masses are always desirable. Hence, instead of using previous electromechanical types of deploying systems, recent efforts of NASA has concentrated on the use of inflatable/deployable structures for space applications. ${ }^{1}$ Inflatable/deployable membranes have been used in space as parabolic antennas, 
radiators, solar concentrators, sun shields, habitats, radio-frequency structures, optical communication systems, radars, lightweight radiometers, telescopes, etc. Large balloons are also membrane structures that have been used for many scientific missions.

Materials for membrane structures are usually required to have (1) low weight $\left(<0.2 \mathrm{~kg} / \mathrm{m}^{2}\right)$, (2) high tensile strength, (3) high tear strength, (4) low Helium permeability, (5) appropriate thermal properties, (6) low crease sensitivity, (7) seam strength greater than or equal to $80 \%$ of the parent material strength, and (8) no significant losses in tensile or peal strength at low temperature (e.g., $\left.-30^{\circ} \mathrm{C}\right) .^{2,3}$ Materials for current scientific membrane structures include reinforced films and coated fabrics. The reinforced films are generally polyethylene, polyester (e.g., Mylar), and nylon; and the coated fabrics are typically nylon or polyester fabric coated with silicone, polyethylene, polyurethane, polyester, or nylon. ${ }^{2}$ The majority of these materials are being developed by racing yacht sails, packaging, geomembrane, parachute, and textile industries. The advantages of membrane structures include small stowed volume, light weight, low cost, and good thermal and damping properties. ${ }^{4,5}$ However, there are difficulties in the design of large scientific membranes. ${ }^{6}$

A few factors need to be considered in designing large membrane structures, which include construction of smooth membrane surfaces using gores and seam tapes, minimum folding requirement for stowage, deployability, accuracy of the deployed shape, positive principle stresses to prevent wrinkling, large principle stresses to provide enough global bending stiffness, small principle stresses to prevent material creeping and change of the deployed shape, and easy manufacturing. These are complex, stringent, and somewhat conflicting design requirements. Hence, there are unique issues that need to be solved in order to assess and satisfy these design requirements.

\subsection{Modeling issues}

Membranes are two-dimensional (2D) analogy of cables, and they have thin thickness and negligible bending stiffness. Hence, membranes support loads mainly by in-plane stretching and, sometimes, shearing. Since stretching is the main load path, thickness change due to Poisson's effect can be significant. For a material with a Poisson ratio close to 0.5 (e.g., rubber), the material stiffness matrix is singular if the thickness change due to Poisson's effect is neglected. Moreover, membranes are usually designed for undergoing large displacements and rotations but not for small or even large strains during regular operations. ${ }^{2}$ Large rotations cause geometric nonlinearities and make some strain and stress measures nonobjective. ${ }^{7}$ Hence, choosing an appropriate strain measure that excludes rigid-body displacements and rotations becomes the major factor in deriving a fully nonlinear membrane theory.

Wrinkling is a local buckling phenomenon of $2 \mathrm{D}$ structures with negligible bending stiffness. A membrane theory without accounting for bending stiffness 
predicts that wrinkling will happen when the smallest in-plane principal stress decreases from positive to negative. On the other hand, a membrane theory accounting for bending stiffness predicts that wrinkling will happen only when the local tangent stiffness decreases from positive to negative, which usually corresponds to a negative but small principal stress. Furthermore, only a membrane theory with bending stiffness can be used for post-buckling analyses to predict the bifurcated deformation shapes after wrinkling. Although rolling rather than folding is usually preferred in packing inflatable structures, ${ }^{1}$ folding is necessary in many cases. Unfortunately, folding may create permanent folding lines in crease sensitive membranes, and such folding lines cause surface distortion of the inflated membranes. ${ }^{8}$ To study the influences of folding lines requires the use of a membrane theory that accounts for bending stiffness.

Although the inflated shape of a membrane can be designed by varying the distribution of membrane thickness, research shows that temperature change may degrade prediction accuracy much more severely than thickness variations. ${ }^{9}$

Hence, a membrane theory for analyzing scientific membranes should account for geometric nonlinearities due to large rotations, thickness change due to Poisson's effect, bending stiffness, and thermal influences.

\subsection{Analysis issues}

Analysis of scientific membrane structures is required with an accuracy beyond the customary tolerances of conventional structural engineering. For example, a shape prediction tolerance of $0.1 \mathrm{~mm}$ for a radio-frequency membrane reflector having a diameter of a few meters may be taken as the required accuracy of analytical solutions. ${ }^{9,10}$

As membrane materials are usually viscoelastic, membrane structures are designed to have small principle stresses in order to reduce the possibility of creep. However, a membrane with small stresses is vulnerable to wrinkling when the structure is subjected to external localized disturbing loads. Because of the nonlinearities and multiple loading and unloading paths, the wrinkles may stay even after the disturbing loads are released. On the other hand, because the magnitudes of internal stresses determine the global bending stiffnesses of a membrane, the principle stresses should be designed to be positive and as large as creep concerns will allow.

Although creep is less of a problem for membranes because the internal pressure gradient can be adjusted and hence creep can be viewed as a change in the initial shape. Nonuniform creep due to nonuniform stresses may cause the deployed shape to deviate from the required one even after makeup gas is replenished. Hence, it is better to design a membrane to have uniform internal stresses after deployment.

The change of deployed shape and redistribution of loads and load carrying capabilities of a membrane after wrinkling need to be understood in order to design accurate and durable membranes, which requires complex stability and bifurcation 
analyses. Moreover, because membranes may experience strain values as large as $14 \%$ before burst $^{2}$ and membrane materials may be viscoelastic, their material properties may depend on strains and/or strain rates and need to be obtained experimentally before design analysis.

Although an initially flat membrane can be inflated to approximate the geometry of a parabolic antenna, the inflation pressure required is so high that the makeup gas needed to replenish possible loss of gas due to meteoroid damage is also high and hence heavy. ${ }^{11}$ Hence, it is better to use an initially curved membrane to design an accurate deployed shape with a low inflation pressure. However, the design problem becomes how to find an unknown undeformed membrane geometry with unknown thickness distribution that will result in the required deployed shape with a known inflation pressure, which is a challenging nonlinear inverse problem.

In the past, space systems experienced high failure rates during deployment but usually performed well after deployment. ${ }^{1}$ With inflatable structures, the stake is even higher because their load carrying ability is commensurable with their state of deployment. To prevent deployment failure, simulation of the transient dynamics of inflatable structures is needed, which requires advanced modeling and analysis techniques.

The above discussions show that designing scientific membranes is challenging because of geometric nonlinearities, accuracy requirement, viscoelastic material properties, creep, wrinkling, inverse design problems, and transient deployment dynamics. However, an accurate nonlinear membrane theory plays the key role in solving these problems.

\subsection{Objective}

The objective is to derive a fully nonlinear membrane theory that can be used for accurate nonlinear analysis of precision membranes. Moreover, because rotationally symmetric membranes are popular in scientific applications, nonlinear ODSs governing their static deformations under axisymmetric loading will be listed and a numerical method for investigating their load-deflection characteristics will be derived and numerically verified. Furthermore, a method for computing the unknown undeformed membrane geometry that is needed for manufacturing from the known/required inflated geometry and the known/designed inflation pressure will be derived and numerically verified.

\section{Fully Nonlinear Membrane Theory}

Membranes as well as plates and shells are 2D (two-dimensional) structures. An initially curved membrane is a thin shell with negligible bending and twisting stiffnesses. Hence, we first treat a membrane as a doubly-curved shell in deriving a fully nonlinear membrane theory for general use, and then we apply assumptions to obtain governing equations for simplified types of membranes, especially for thin 


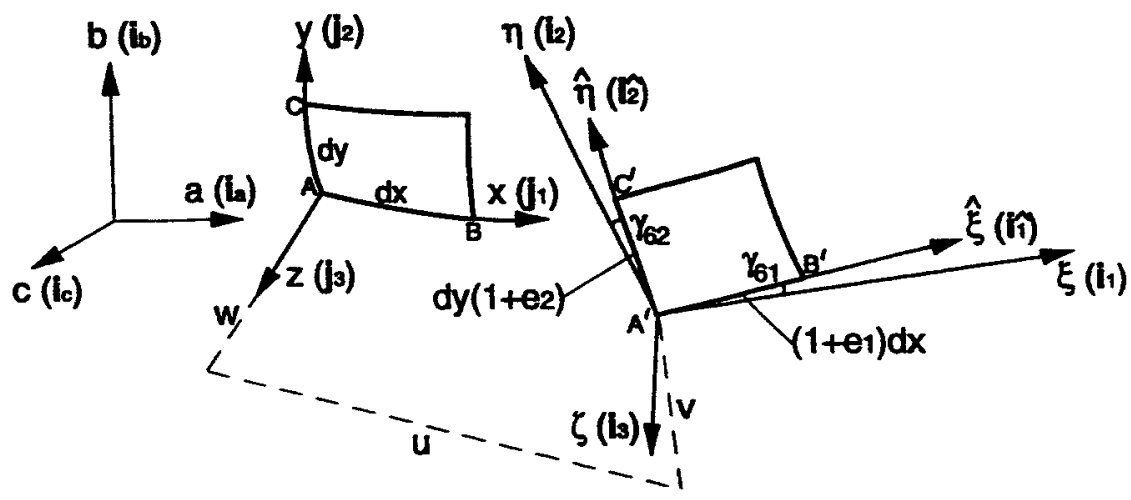

Fig. 1. The undeformed and deformed geometries of a differential reference surface and the three coordinate systems used.

axisymmetric membranes. The two major tasks in deriving a nonlinear 2D structural theory are (1) how to describe the cross section warping, and (2) how to describe the reference surface deformation. Different approximations of the cross section warping result in different shear deformation theories, and different approximations of the reference surface deformation result in different geometrically nonlinear theories. As membranes have very thin thickness, the strain energy due to cross section warping is negligible. Next we present geometrically exact descriptions of the undeformed and deformed geometries of an initially curved surface.

\subsection{Surface analysis}

\subsubsection{Coordinate systems and curvatures}

Two coordinate systems are required in order to describe the deformation of an initially curved 2D structures: one describes the undeformed reference surface and the other describes the deformed reference surface. Figure 1 shows an infinitesimal area of the reference surface of a 2D structure before and after deformation. The $x y z$ is an orthogonal curvilinear coordinate system with the $x$ and $y$ being curvilinear axes on the undeformed reference surface and the $z$ being a rectilinear axis, and the $\xi \eta \zeta$ is an orthogonal curvilinear coordinate system with the $\xi$ and $\eta$ being curvilinear axes on the deformed reference surface and the $\zeta$ being a rectilinear axis. An inertial rectangular coordinate system $a b c$ is also used, for reference purpose, in the calculation of initial curvatures. We let $\mathbf{j}_{1}, \mathbf{j}_{2}$, and $\mathbf{j}_{3}$ denote the unit vectors along the axes $x, y$, and $z ; \mathbf{i}_{1}, \mathbf{i}_{2}$, and $\mathbf{i}_{3}$ denote the unit vectors along the axes $\xi$, $\eta$, and $\zeta ;$ and $\mathbf{i}_{a}, \mathbf{i}_{b}$, and $\mathbf{i}_{c}$ denote the unit vectors along the axes $a, b$, and $c$.

To obtain initial curvatures, the undeformed position vector $\mathbf{P}$ of point $A$ is presented as ${ }^{12}$ :

$$
\mathbf{P}=P_{1}(x, y) \mathbf{i}_{a}+P_{2}(x, y) \mathbf{i}_{b}+P_{3}(x, y) \mathbf{i}_{c} .
$$


Taking first- and second-order derivatives of Eq. (1) with respect to $x$ and $y$ yields

$$
\begin{aligned}
\mathbf{j}_{1} & =\frac{\partial \mathbf{P}}{\partial x}=P_{1 x} \mathbf{i}_{a}+P_{2 x} \mathbf{i}_{b}+P_{3 x} \mathbf{i}_{c} \\
\mathbf{j}_{2} & =\frac{\partial \mathbf{P}}{\partial y}=P_{1 y} \mathbf{i}_{a}+P_{2 y} \mathbf{i}_{b}+P_{3 y} \mathbf{i}_{c} \\
\mathbf{j}_{3} & =\mathbf{j}_{1} \times \mathbf{j}_{2} \\
& =\left(P_{2 x} P_{3 y}-P_{3 x} P_{2 y}\right) \mathbf{i}_{a}+\left(P_{3 x} P_{1 y}-P_{1 x} P_{3 y}\right) \mathbf{i}_{b}+\left(P_{1 x} P_{2 y}-P_{2 x} P_{1 y}\right) \mathbf{i}_{c}
\end{aligned}
$$

and

$$
\begin{aligned}
& \frac{\partial\left\{\mathbf{j}_{123}\right\}}{\partial x}=\left[K_{1}^{0}\right]\left\{\mathbf{j}_{123}\right\}, \quad\left[K_{1}^{0}\right] \equiv\left[\begin{array}{ccc}
0 & k_{5}^{0} & -k_{1}^{0} \\
-k_{5}^{0} & 0 & -k_{61}^{0} \\
k_{1}^{0} & k_{61}^{0} & 0
\end{array}\right], \\
& \frac{\partial\left\{\mathbf{j}_{123}\right\}}{\partial y}=\left[K_{2}^{0}\right]\left\{\mathbf{j}_{123}\right\}, \quad\left[K_{2}^{0}\right] \equiv\left[\begin{array}{ccc}
0 & k_{4}^{0} & -k_{62}^{0} \\
-k_{4}^{0} & 0 & -k_{2}^{0} \\
k_{62}^{0} & k_{2}^{0} & 0
\end{array}\right],
\end{aligned}
$$

where ()$_{x} \equiv \partial() / \partial x,()_{y} \equiv \partial() / \partial y,\left\{\mathbf{j}_{123}\right\} \equiv\left\{\mathbf{j}_{1}, \mathbf{j}_{2}, \mathbf{j}_{3}\right\}^{T}$, and $\left[K_{1}^{0}\right]$ and $\left[K_{2}^{0}\right]$ are initial curvature matrices. $k_{1}^{0}$ and $k_{2}^{0}$ are curvatures of bending with respect to the axes $y$ and $-x$, respectively. $k_{61}^{0}$ and $k_{62}^{0}$ are curvatures of twisting with respect to the axes $-x$ and $y$, respectively. $k_{4}^{0}$ is the spiral curvature of the $y$ axis with respect to the $z$ axis; and $k_{5}^{0}$ is the spiral curvature of the $x$ axis with respect to the $z$ axis. The initial curvatures can be derived using Eq. (3). For example,

$$
\begin{aligned}
k_{1}^{0} \equiv & -\frac{\partial \mathbf{j}_{1}}{\partial x} \cdot \mathbf{j}_{3} \\
= & -P_{1 x x}\left(P_{2 x} P_{3 y}-P_{3 x} P_{2 y}\right)-P_{2 x x}\left(P_{3 x} P_{1 y}-P_{1 x} P_{3 y}\right) \\
& -P_{3 x x}\left(P_{1 x} P_{2 y}-P_{2 x} P_{1 y}\right) .
\end{aligned}
$$

In Fig. 1, $u, v$, and $w$ are the displacements of point $\mathrm{A}$ with respect to the axes $x$, $y$, and $z$, respectively. The axes $\hat{\xi}$ and $\hat{\eta}$ represent the convected configurations of the axes $x$ and $y$, respectively; and $\gamma_{6}\left(=\gamma_{61}+\gamma_{62}\right)$ is the in-plane shear deformation. We let $\mathbf{i}_{\hat{1}}$ and $\mathbf{i}_{\hat{2}}$ denote the unit vectors along the axes $\hat{\xi}$ and $\hat{\eta}$, respectively. We note that the axes $\xi$ and $\eta$ coincide with the axes $\hat{\xi}$ and $\hat{\eta}$ only if the in-plane shear deformation $\gamma_{6}$ is zero. Letting $\mathbf{D} \equiv \mathbf{A} \mathbf{A}^{\prime}=u \mathbf{j}_{1}+v \mathbf{j}_{2}+w \mathbf{j}_{3}$ and using Eq. (3), we obtain

$$
\begin{aligned}
\mathbf{A}^{\prime} \mathbf{B}^{\prime}= & -\mathbf{D}+d x \mathbf{j}_{1}+\mathbf{D}+\frac{\partial \mathbf{D}}{\partial x} d x=d x \mathbf{j}_{1}+\frac{\partial \mathbf{D}}{\partial x} d x \\
= & {\left[\left(1+u_{x}-v k_{5}^{0}+w k_{1}^{0}\right) \mathbf{j}_{1}+\left(v_{x}+u k_{5}^{0}+w k_{61}^{0}\right) \mathbf{j}_{2}\right.} \\
& \left.+\left(w_{x}-u k_{1}^{0}-v k_{61}^{0}\right) \mathbf{j}_{3}\right] d x
\end{aligned}
$$




$$
\begin{aligned}
\mathbf{A}^{\prime} \mathbf{C}^{\prime}= & -\mathbf{D}+d y \mathbf{j}_{2}+\mathbf{D}+\frac{\partial \mathbf{D}}{\partial y} d y=d y \mathbf{j}_{2}+\frac{\partial \mathbf{D}}{\partial y} d y \\
= & {\left[\left(u_{y}-v k_{4}^{0}+w k_{62}^{0}\right) \mathbf{j}_{1}+\left(1+v_{y}+u k_{4}^{0}+w k_{2}^{0}\right) \mathbf{j}_{2}\right.} \\
& \left.+\left(w_{y}-u k_{62}^{0}-v k_{2}^{0}\right) \mathbf{j}_{3}\right] d y .
\end{aligned}
$$

Hence, the axial strains along the axes $\hat{\xi}$ and $\hat{\eta}, e_{1}$ and $e_{2}$, are given by:

$$
\begin{aligned}
e_{1}= & \frac{\overline{A^{\prime} B^{\prime}}-d x}{d x} \\
= & {\left[\left(1+u_{x}-v k_{5}^{0}+w k_{1}^{0}\right)^{2}+\left(v_{x}+u k_{5}^{0}+w k_{61}^{0}\right)^{2}\right.} \\
& \left.+\left(w_{x}-u k_{1}^{0}-v k_{61}^{0}\right)^{2}\right]^{1 / 2}-1, \\
e_{2}= & \frac{\overline{A^{\prime} C^{\prime}}-d y}{d y} \\
= & {\left[\left(u_{y}-v k_{4}^{0}+w k_{62}^{0}\right)^{2}+\left(1+v_{y}+u k_{4}^{0}+w k_{2}^{0}\right)^{2}\right.} \\
& \left.+\left(w_{y}-u k_{62}^{0}-v k_{2}^{0}\right)^{2}\right]^{1 / 2}-1 .
\end{aligned}
$$

The unit vectors along the $\hat{\xi}$ and $\hat{\eta}$ directions are given by:

$$
\begin{aligned}
& \mathbf{i}_{\hat{\mathbf{i}}}=\frac{\mathbf{A}^{\prime} \mathbf{B}^{\prime}}{\left(1+e_{1}\right) d x}=\hat{T}_{11} \mathbf{j}_{1}+\hat{T}_{12} \mathbf{j}_{2}+\hat{T}_{13} \mathbf{j}_{\mathbf{3}}, \\
& \mathbf{i}_{\hat{\mathbf{2}}}=\frac{\mathbf{A}^{\prime} \mathbf{C}^{\prime}}{\left(1+e_{2}\right) d y}=\hat{T}_{21} \mathbf{j}_{1}+\hat{T}_{22} \mathbf{j}_{2}+\hat{T}_{23} \mathbf{j}_{3},
\end{aligned}
$$

where

$$
\begin{gathered}
\hat{T}_{11}=\frac{1+u_{x}-v k_{5}^{0}+w k_{1}^{0}}{1+e_{1}}, \quad \hat{T}_{12}=\frac{v_{x}+u k_{5}^{0}+w k_{61}^{0}}{1+e_{1}} \\
\hat{T}_{13}=\frac{w_{x}-u k_{1}^{0}-v k_{61}^{0}}{1+e_{1}}, \quad \hat{T}_{21}=\frac{u_{y}-v k_{4}^{0}+w k_{62}^{0}}{1+e_{2}} \\
\hat{T}_{22}=\frac{1+v_{y}+u k_{4}^{0}+w k_{2}^{0}}{1+e_{2}}, \quad \hat{T}_{23}=\frac{w_{y}-u k_{62}^{0}-v k_{2}^{0}}{1+e_{2}}
\end{gathered}
$$

It follows from Fig. 1 and Eqs. (8) and (9) that

$$
\gamma_{6} \equiv \gamma_{61}+\gamma_{62}=\sin ^{-1}\left(\mathbf{i}_{\hat{1}} \cdot \mathbf{i}_{\hat{2}}\right)=\sin ^{-1}\left(\hat{T}_{11} \hat{T}_{21}+\hat{T}_{12} \hat{T}_{22}+\hat{T}_{13} \hat{T}_{23}\right) \text {. }
$$

Hence, $\gamma_{6}$ can be represented in terms of $u, v$, and $w$. Moreover, one can use the symmetry of Jaumann shear strains to prove that ${ }^{7}$

$$
\left(1+e_{1}\right) \sin \gamma_{61}=\left(1+e_{2}\right) \sin \gamma_{62} \text {. }
$$

Then, the unique expressions of $\gamma_{61}$ and $\gamma_{62}$ can be determined from Eqs. (11a) and (11b). The unit vector normal to the deformed reference surface, $\mathbf{i}_{3}$, is given by:

$$
\mathbf{i}_{3}=\frac{\mathbf{i}_{\hat{1}} \times \mathbf{i}_{\hat{\mathbf{2}}}}{\left|\mathbf{i}_{\hat{\mathbf{1}}} \times \mathbf{i}_{\hat{2}}\right|}=T_{31} \mathbf{j}_{1}+T_{32} \mathbf{j}_{2}+T_{33} \mathbf{j}_{3},
$$


where

$$
\begin{aligned}
T_{31} & =\frac{\hat{T}_{12} \hat{T}_{23}-\hat{T}_{13} \hat{T}_{22}}{R_{0}}, \quad T_{32}=\frac{\hat{T}_{13} \hat{T}_{21}-\hat{T}_{11} \hat{T}_{23}}{R_{0}} \\
T_{33} & =\frac{\hat{T}_{11} \hat{T}_{22}-\hat{T}_{12} \hat{T}_{21}}{R_{0}} \\
R_{0} & \equiv\left[\left(\hat{T}_{12} \hat{T}_{23}-\hat{T}_{13} \hat{T}_{22}\right)^{2}+\left(\hat{T}_{13} \hat{T}_{21}-\hat{T}_{11} \hat{T}_{23}\right)^{2}+\left(\hat{T}_{11} \hat{T}_{22}-\hat{T}_{12} \hat{T}_{21}\right)^{2}\right]^{1 / 2} \\
& =\left|\cos \gamma_{6}\right| .
\end{aligned}
$$

Using Fig. 1 and Eqs. (8), (9), and (12), we obtain the following transformation which relates the undeformed coordinate system $x y z$ to the deformed coordinate system $\xi \eta \zeta$ :

$$
\left\{\mathbf{i}_{123}\right\}=[T]\left\{\mathbf{j}_{123}\right\},[T] \equiv[\Gamma]\left[\begin{array}{lll}
\hat{T}_{11} & \hat{T}_{12} & \hat{T}_{13} \\
\hat{T}_{21} & \hat{T}_{22} & \hat{T}_{23} \\
T_{31} & T_{32} & T_{33}
\end{array}\right]
$$

where

$$
\begin{aligned}
\left\{\mathbf{i}_{123}\right\} & =[\Gamma]\left\{\mathbf{i}_{\hat{1} \hat{2} 3}\right\} \\
{[\Gamma] } & \equiv\left[\begin{array}{ccc}
\cos \gamma_{61} & \sin \gamma_{61} & 0 \\
\sin \gamma_{62} & \cos \gamma_{62} & 0 \\
0 & 0 & 1
\end{array}\right]^{-1} \\
& =\frac{1}{\cos \gamma_{6}}\left[\begin{array}{ccc}
\cos \gamma_{62} & -\sin \gamma_{61} & 0 \\
-\sin \gamma_{62} & \cos \gamma_{61} & 0 \\
0 & 0 & \cos \gamma_{6}
\end{array}\right] .
\end{aligned}
$$

$\left\{\mathbf{i}_{123}\right\} \equiv\left\{\mathbf{i}_{1}, \mathbf{i}_{2}, \mathbf{i}_{3}\right\}^{T}$, and $\left\{\mathbf{i}_{\hat{1} \hat{2} 3}\right\} \equiv\left\{\mathbf{i}_{\hat{1}}, \mathbf{i}_{\hat{2}}, \mathbf{i}_{3}\right\}^{T}$. Moreover, using Eqs. (14a) and (3) and the following identities

$$
\begin{gathered}
\frac{\partial \mathbf{i}_{j}}{\partial x} \cdot \mathbf{i}_{j}=\frac{\partial \mathbf{i}_{j}}{\partial y} \cdot \mathbf{i}_{j}=0, \quad \frac{\partial \mathbf{i}_{j}}{\partial x} \cdot \mathbf{i}_{k}=-\frac{\partial \mathbf{i}_{k}}{\partial x} \cdot \mathbf{i}_{j} \\
\frac{\partial \mathbf{i}_{j}}{\partial y} \cdot \mathbf{i}_{k}=-\frac{\partial \mathbf{i}_{k}}{\partial y} \cdot \mathbf{i}_{j}, \quad[T]^{-1}=[T]^{T}
\end{gathered}
$$

we obtain

$$
\begin{aligned}
\frac{\partial\left\{\mathbf{i}_{123}\right\}}{\partial x} & =\left[K_{1}\right]\left\{\mathbf{i}_{123}\right\} \\
{\left[K_{1}\right] } & \equiv\left[\begin{array}{ccc}
0 & k_{5} & -k_{1} \\
-k_{5} & 0 & -k_{61} \\
k_{1} & k_{61} & 0
\end{array}\right]=\frac{\partial[T]}{\partial x}[T]^{T}+[T]\left[K_{1}^{0}\right][T]^{T},
\end{aligned}
$$




$$
\begin{aligned}
\frac{\partial\left\{\mathbf{i}_{123}\right\}}{\partial y} & =\left[K_{2}\right]\left\{\mathbf{i}_{123}\right\} \\
{\left[K_{2}\right] \equiv } & {\left[\begin{array}{ccc}
0 & k_{4} & -k_{62} \\
-k_{4} & 0 & -k_{2} \\
k_{62} & k_{2} & 0
\end{array}\right]=\frac{\partial[T]}{\partial y}[T]^{T}+[T]\left[K_{2}^{0}\right][T]^{T} }
\end{aligned}
$$

where $\left[K_{1}\right]$ and $\left[K_{2}\right]$ are called deformed curvature matrices in this work and

$$
\begin{gathered}
k_{1} \equiv-\frac{\partial \mathbf{i}_{1}}{\partial x} \cdot \mathbf{i}_{3}=-T_{1 m x} T_{3 m}-T_{21} k_{61}^{0}+T_{22} k_{1}^{0}+T_{23} k_{5}^{0}, \\
k_{2} \equiv-\frac{\partial \mathbf{i}_{2}}{\partial y} \cdot \mathbf{i}_{3}=-T_{2 m y} T_{3 m}+T_{11} k_{2}^{0}-T_{12} k_{62}^{0}-T_{13} k_{4}^{0} \\
k_{61} \equiv-\frac{\partial \mathbf{i}_{2}}{\partial x} \cdot \mathbf{i}_{3}=-T_{2 m x} T_{3 m}+T_{11} k_{61}^{0}-T_{12} k_{1}^{0}-T_{13} k_{5}^{0}, \\
k_{62} \equiv-\frac{\partial \mathbf{i}_{1}}{\partial y} \cdot \mathbf{i}_{3}=-T_{1 m y} T_{3 m}-T_{21} k_{2}^{0}+T_{22} k_{62}^{0}+T_{23} k_{4}^{0}, \\
k_{5} \equiv \frac{\partial \mathbf{i}_{1}}{\partial x} \cdot \mathbf{i}_{2}=T_{1 m x} T_{2 m}-T_{31} k_{61}^{0}+T_{32} k_{1}^{0}+T_{33} k_{5}^{0}, \\
k_{4} \equiv-\frac{\partial \mathbf{i}_{2}}{\partial y} \cdot \mathbf{i}_{1}=-T_{2 m y} T_{1 m}-T_{31} k_{2}^{0}+T_{32} k_{62}^{0}+T_{33} k_{4}^{0}
\end{gathered}
$$

Here, $\mathbf{i}_{1}=\mathbf{i}_{2} \times \mathbf{i}_{3}, \mathbf{i}_{2}=\mathbf{i}_{3} \times \mathbf{i}_{1}, \mathbf{i}_{3}=\mathbf{i}_{1} \times \mathbf{i}_{2}$, and Eq. (14a) are used. Hereafter, unless otherwise stated, repeated subscript indices imply summations. We note that the $k_{j}$ in Eq. (18) are functions needed for exact description of $\xi$ and $\eta$, but they are not real curvatures because the deformed $d x(d y)$ is not along the $\mathbf{i}_{1}\left(\mathbf{i}_{2}\right)$ direction because $\gamma_{61} \neq 0\left(\gamma_{62} \neq 0\right)$. If $\gamma_{61}=\gamma_{62}=0$, the curvatures are normalized (but not real) curvatures because the differentiations in Eq. (18) are taken with respect to the undeformed lengths $d x$ and $d y$, instead of the deformed lengths $\left(1+e_{1}\right) d x$ and $\left(1+e_{2}\right) d y$. Only if $\gamma_{61}=\gamma_{62}=e_{1}=e_{2}=0, k_{j}$ represent real curvatures.

\subsubsection{Variations of global strains}

To derive the governing equations using the extended Hamilton's principle, we need to have the variations of the global strains $e_{1}, e_{2}, \gamma_{61}, \gamma_{62}$, and $k_{i}$. Taking variations of Eqs. (6) and (7) and using Eq. (10), we obtain the variations of $e_{1}$ and $e_{2}$ as:

$$
\begin{aligned}
& \delta e_{1}=\hat{T}_{11} \delta t_{11}+\hat{T}_{12} \delta t_{12}+\hat{T}_{13} \delta t_{13}, \\
& \delta e_{2}=\hat{T}_{21} \delta t_{21}+\hat{T}_{22} \delta t_{22}+\hat{T}_{23} \delta t_{23},
\end{aligned}
$$


where

$$
\begin{aligned}
& \delta t_{11}=\delta\left(1+u_{x}-v k_{5}^{0}+w k_{1}^{0}\right)=\delta u_{x}-k_{5}^{0} \delta v+k_{1}^{0} \delta w \\
& \delta t_{12}=\delta\left(v_{x}+u k_{5}^{0}+w k_{61}^{0}\right)=\delta v_{x}+k_{5}^{0} \delta u+k_{61}^{0} \delta w \\
& \delta t_{13}=\delta\left(w_{x}-u k_{1}^{0}-v k_{61}^{0}\right)=\delta w_{x}-k_{1}^{0} \delta u-k_{61}^{0} \delta v \\
& \delta t_{21}=\delta\left(u_{y}-v k_{4}^{0}+w k_{62}^{0}\right)=\delta u_{y}-k_{4}^{0} \delta v+k_{62}^{0} \delta w \\
& \delta t_{22}=\delta\left(1+v_{y}+u k_{4}^{0}+w k_{2}^{0}\right)=\delta v_{y}+k_{4}^{0} \delta u+k_{2}^{0} \delta w \\
& \delta t_{23}=\delta\left(w_{y}-u k_{62}^{0}-v k_{2}^{0}\right)=\delta w_{y}-k_{62}^{0} \delta u-k_{2}^{0} \delta v
\end{aligned}
$$

Moreover, taking variations of Eqs. (8) and (9) and using Eq. (10) yields

$$
\begin{aligned}
& \delta \mathbf{i}_{\hat{1}}=\frac{1}{1+e_{1}}\left(\mathbf{j}_{1} \delta t_{11}+\mathbf{j}_{2} \delta t_{12}+\mathbf{j}_{3} \delta t_{13}-\mathbf{i}_{\hat{1}} \delta e_{1}\right), \\
& \delta \mathbf{i}_{\hat{2}}=\frac{1}{1+e_{2}}\left(\mathbf{j}_{1} \delta t_{21}+\mathbf{j}_{2} \delta t_{22}+\mathbf{j}_{3} \delta t_{23}-\mathbf{i}_{\hat{2}} \delta e_{2}\right) .
\end{aligned}
$$

Taking the variation of $\sin \gamma_{6}=\mathbf{i}_{\hat{1}} \cdot \mathbf{i}_{2}$ (see Eq. (11a)) and using Eqs. (22), (8), (9), (19), and (20), we obtain

$$
\begin{aligned}
\delta \gamma_{6}= & \frac{\left(\hat{T}_{21}-\sin \gamma_{6} \hat{T}_{11}\right) \delta t_{11}+\left(\hat{T}_{22}-\sin \gamma_{6} \hat{T}_{12}\right) \delta t_{12}+\left(\hat{T}_{23}-\sin \gamma_{6} \hat{T}_{13}\right) \delta t_{13}}{\cos \gamma_{6}\left(1+e_{1}\right)} \\
& +\frac{\left(\hat{T}_{11}-\sin \gamma_{6} \hat{T}_{21}\right) \delta t_{21}+\left(\hat{T}_{12}-\sin \gamma_{6} \hat{T}_{22}\right) \delta t_{22}+\left(\hat{T}_{13}-\sin \gamma_{6} \hat{T}_{23}\right) \delta t_{23}}{\cos \gamma_{6}\left(1+e_{2}\right)} .
\end{aligned}
$$

Taking the variation of Eq. (11b) and using the fact that $\delta \gamma_{6}=\delta \gamma_{61}+\delta \gamma_{62}$, we obtain

$$
\begin{aligned}
& \delta \gamma_{61}=\frac{\left(1+e_{2}\right) \cos \gamma_{62} \delta \gamma_{6}-\sin \gamma_{61} \delta e_{1}+\sin \gamma_{62} \delta e_{2}}{\left(1+e_{1}\right) \cos \gamma_{61}+\left(1+e_{2}\right) \cos \gamma_{62}}, \\
& \delta \gamma_{62}=\frac{\left(1+e_{1}\right) \cos \gamma_{61} \delta \gamma_{6}+\sin \gamma_{61} \delta e_{1}-\sin \gamma_{62} \delta e_{2}}{\left(1+e_{1}\right) \cos \gamma_{61}+\left(1+e_{2}\right) \cos \gamma_{62}},
\end{aligned}
$$

where $\delta \gamma_{6}$ is shown in Eq. (23).

To obtain the variations of curvatures (i.e., $\delta k_{j}$ ), the concept of orthogonal virtual rotations is needed. Because the variations of the unit vectors $\mathbf{i}_{k}$ are due to the virtual rotations of the observed membrane element, we have

$$
\delta\left\{\mathbf{i}_{123}\right\}=[\delta \theta]\left\{\mathbf{i}_{123}\right\}, \quad[\delta \theta] \equiv\left[\begin{array}{ccc}
0 & \delta \theta_{3} & -\delta \theta_{2} \\
-\delta \theta_{3} & 0 & \delta \theta_{1} \\
\delta \theta_{2} & -\delta \theta_{1} & 0
\end{array}\right]
$$


where $\delta \theta_{1}, \delta \theta_{2}$, and $\delta \theta_{3}$ are the virtual rigid-body rotations of the observed membrane element with respect to the axes $\xi, \eta$, and $\zeta$, respectively. We note that $\delta \theta_{i}$ are differential rotations and hence they are vector quantities. Moreover, $\delta \theta_{i}$ are along three perpendicular directions and hence they are mutually independent. Taking the variations of curvatures defined in Eq. (18) and using Eqs. (16), (17), and (25), one can obtain that ${ }^{12}$

$$
\begin{aligned}
& \left\{\begin{array}{c}
-\delta k_{61} \\
\delta k_{1} \\
\delta k_{5}
\end{array}\right\}=\frac{\partial}{\partial x}\left\{\begin{array}{c}
\delta \theta_{1} \\
\delta \theta_{2} \\
\delta \theta_{3}
\end{array}\right\}-\left[K_{1}\right]\left\{\begin{array}{c}
\delta \theta_{1} \\
\delta \theta_{2} \\
\delta \theta_{3}
\end{array}\right\}, \\
& \left\{\begin{array}{c}
-\delta k_{2} \\
\delta k_{62} \\
\delta k_{4}
\end{array}\right\}=\frac{\partial}{\partial y}\left\{\begin{array}{c}
\delta \theta_{1} \\
\delta \theta_{2} \\
\delta \theta_{3}
\end{array}\right\}-\left[K_{2}\right]\left\{\begin{array}{c}
\delta \theta_{1} \\
\delta \theta_{2} \\
\delta \theta_{3}
\end{array}\right\} .
\end{aligned}
$$

Using Eqs. (25), (14b), and (22) and the fact that $\mathbf{i}_{3} \cdot \mathbf{i}_{\hat{1}}=\mathbf{i}_{3} \cdot \mathbf{i}_{\hat{2}}=0$, we obtain that

$$
\begin{aligned}
\delta \theta_{1}= & \delta \mathbf{i}_{2} \cdot \mathbf{i}_{3}=\frac{\cos \gamma_{61}}{\cos \gamma_{6}} \delta \mathbf{i}_{\hat{2}} \cdot \mathbf{i}_{3}-\frac{\sin \gamma_{62}}{\cos \gamma_{6}} \delta \mathbf{i}_{\hat{\mathbf{i}}} \cdot \mathbf{i}_{3} \\
= & \frac{\cos \gamma_{61}}{\cos \gamma_{6}\left(1+e_{2}\right)}\left(T_{31} \delta t_{21}+T_{32} \delta t_{22}+T_{33} \delta t_{23}\right) \\
& -\frac{\sin \gamma_{62}}{\cos \gamma_{6}\left(1+e_{1}\right)}\left(T_{31} \delta t_{11}+T_{32} \delta t_{12}+T_{33} \delta t_{13}\right), \\
\delta \theta_{2}= & -\delta \mathbf{i}_{1} \cdot \mathbf{i}_{3}=\frac{\sin \gamma_{61}}{\cos \gamma_{6}} \delta \mathbf{i}_{\hat{2}} \cdot \mathbf{i}_{3}-\frac{\cos \gamma_{62}}{\cos \gamma_{6}} \delta \mathbf{i}_{\hat{1}} \cdot \mathbf{i}_{3} \\
= & \frac{\sin \gamma_{61}}{\cos \gamma_{6}\left(1+e_{2}\right)}\left(T_{31} \delta t_{21}+T_{32} \delta t_{22}+T_{33} \delta t_{23}\right) \\
& -\frac{\cos \gamma_{62}}{\cos \gamma_{6}\left(1+e_{1}\right)}\left(T_{31} \delta t_{11}+T_{32} \delta t_{12}+T_{33} \delta t_{13}\right) .
\end{aligned}
$$

Using Eqs. (25), (14b), and (22), we obtain that

$$
\begin{aligned}
\delta \theta_{3}= & \frac{1}{2}\left(\delta \mathbf{i}_{1} \cdot \mathbf{i}_{2}-\delta \mathbf{i}_{2} \cdot \mathbf{i}_{1}\right)=\frac{1}{2}\left(\delta \gamma_{62}-\delta \gamma_{61}\right)+\frac{1}{2 \cos \gamma_{6}}\left(\delta \mathbf{i}_{\hat{1}} \cdot \mathbf{i}_{\hat{2}}-\delta \mathbf{i}_{\hat{2}} \cdot \mathbf{i}_{\hat{1}}\right) \\
= & \frac{1}{2}\left(\delta \gamma_{62}-\delta \gamma_{61}\right) \\
& +\frac{\left(\hat{T}_{21}-\sin \gamma_{6} \hat{T}_{11}\right) \delta t_{11}+\left(\hat{T}_{22}-\sin \gamma_{6} \hat{T}_{12}\right) \delta t_{12}+\left(\hat{T}_{23}-\sin \gamma_{6} \hat{T}_{13}\right) \delta t_{13}}{2 \cos \gamma_{6}\left(1+e_{1}\right)} \\
& -\frac{\left(\hat{T}_{11}-\sin \gamma_{6} \hat{T}_{21}\right) \delta t_{21}+\left(\hat{T}_{12}-\sin \gamma_{6} \hat{T}_{22}\right) \delta t_{22}+\left(\hat{T}_{13}-\sin \gamma_{6} \hat{T}_{23}\right) \delta t_{23}}{2 \cos \gamma_{6}\left(1+e_{2}\right)} .
\end{aligned}
$$


Hence, $\delta e_{1}, \delta e_{2}, \delta \gamma_{6}, \delta \gamma_{61}, \delta \gamma_{62}, \delta \theta_{1}, \delta \theta_{2}$, and $\delta \theta_{3}$ can be represented in terms of $\delta u, \delta v, \delta w, \delta u_{x}, \delta v_{x}, \delta w_{x}, \delta u_{y}, \delta v_{y}$, and $\delta w_{y}$. Moreover, the variations of curvatures $\delta k_{j}$ can be represented in terms of $\delta u, \delta v, \delta w, \delta u_{x}, \delta v_{x}, \delta w_{x}, \delta u_{y}, \delta v_{y}, \delta w_{y}, \delta u_{x x}$, $\delta v_{x x}, \delta w_{x x}, \delta u_{y y}, \delta v_{y y}, \delta w_{y y}, \delta u_{x y}, \delta v_{x y}$, and $\delta w_{x y}$.

\subsection{Jaumann strains and stresses}

Although membranes have thin thickness, it is necessary to account for bending stiffness in order to study wrinkling problems and shape control of inflatable membranes. For the modeling of highly flexible structures, Jaumann strains and stresses are appropriate and convenient measures because they are objective, workconjugate, and fully nonlinear. ${ }^{7}$ Moreover, because Jaumann strains are corotated engineering strains, material properties obtained from experiments using small engineering strains and stresses can be directly applied.

Next we derive the fully nonlinear expressions of Jaumann strains by using the polar decomposition theory and the concept of local relative displacements.

\subsubsection{Polar decomposition}

For an arbitrary point $Q$ on a differential membrane element that has dimensions $d x \times d y \times h$ ( $h=$ thickness) and crosses the reference surface at point A shown in Fig. 1, because transverse shear warping is assumed to be negligible, the undeformed position vector $\mathbf{P}_{\boldsymbol{Q}}$ and the deformed position vector $\mathbf{P}_{\boldsymbol{q}}$ are given by:

$$
\begin{gathered}
\mathbf{P}_{Q}=x \mathbf{j}_{1}+y \mathbf{j}_{2}+z \mathbf{j}_{3}, \\
\mathbf{P}_{q}=(x+u) \mathbf{j}_{1}+(y+v) \mathbf{j}_{2}+w \mathbf{j}_{3}+z\left(1+e_{3}\right) \mathbf{i}_{3}
\end{gathered}
$$

where $e_{3}$ is the extensional strain along the thickness direction and is assumed to be independent of $z$. Because $x$ and $y$ are local curvilinear axes starting from point $\mathrm{A}$,

$$
x=y=0 \text {. }
$$

Taking derivatives of Eq. (30a) and using Eqs. (3) and (31), we derive a gradient tensor $[\breve{Y}]$ as:

$$
[\breve{Y}]=\left[\breve{Y}_{m n}\right] \equiv\left[\mathbf{j}_{m} \cdot \frac{\partial \mathbf{P}_{Q}}{\partial x_{n}}\right]=\left[\begin{array}{ccc}
1+z k_{1}^{0} & z k_{62}^{0} & 0 \\
z k_{61}^{0} & 1+z k_{2}^{0} & 0 \\
0 & 0 & 1
\end{array}\right]
$$

where $x_{n}(n=1,2,3)$ denote $x, y$, and $z$, respectively. Similarly, taking derivatives of Eq. (30b) and using Eqs. (3), (16), (17), (14a), (31), and (10), we obtain the deformation gradient tensor $[Y]$ as: 


$$
\begin{aligned}
{[Y] } & =\left[Y_{m n}\right] \equiv\left[j_{m} \cdot \frac{\partial P_{q}}{\partial x_{n}}\right] \\
& =\left[\begin{array}{lll}
\hat{e}_{1} \hat{T}_{11}+\hat{z}\left(k_{1} T_{11}+k_{61} T_{21}\right)+z e_{3 x} T_{31} & \hat{e}_{2} \hat{T}_{21}+\hat{z}\left(k_{62} T_{11}+k_{2} T_{21}\right)+z e_{3 y} T_{31} & \hat{e}_{3} T_{31} \\
\hat{e}_{1} \hat{T}_{12}+\hat{z}\left(k_{1} T_{12}+k_{61} T_{22}\right)+z e_{3 x} T_{32} & \hat{e}_{2} \hat{T}_{22}+\hat{z}\left(k_{62} T_{12}+k_{2} T_{22}\right)+z e_{3 y} T_{32} & \hat{e}_{3} T_{32} \\
\hat{e}_{1} \hat{T}_{13}+\hat{z}\left(k_{1} T_{13}+k_{61} T_{23}\right)+z e_{3 x} T_{33} & \hat{e}_{2} \hat{T}_{23}+\hat{z}\left(k_{62} T_{13}+k_{2} T_{23}\right)+z e_{3 y} T_{33} & \hat{e}_{3} T_{33}
\end{array}\right],
\end{aligned}
$$

where $\hat{z} \equiv\left(1+e_{3}\right) z, \hat{e}_{1} \equiv 1+e_{1}, \hat{e}_{2} \equiv 1+e_{2}$, and $\hat{e}_{3} \equiv 1+e_{3}$. It can be shown by using the polar decomposition theory that ${ }^{13}$

$$
[Y]=[T]^{T}[U]
$$

where $[U]$ is the so-called right stretch tensor and should be a symmetric matrix, and $[T]$ accounts for the corotation from the system $x y z$ to the system $\xi \eta \zeta$ as shown by Eq. (14a). Jaumann strain tensor $[B]$ is defined to be

$$
[B] \equiv[U]-[I]
$$

where $[I]$ is a $3 \times 3$ identity matrix. It follows from Eqs. (34), (15), (33), and (14a) and the orthogonality of $\mathbf{i}_{j}$ that

$$
[U]=[T][Y]=\left[\begin{array}{ccc}
\hat{e}_{1} \cos \gamma_{61}+\hat{z} k_{1} & \hat{e}_{2} \sin \gamma_{62}+\hat{z} k_{62} & 0 \\
\hat{e}_{1} \sin \gamma_{61}+\hat{z} k_{61} & \hat{e}_{2} \cos \gamma_{62}+\hat{z} k_{2} & 0 \\
z e_{3 x} & z e_{3 y} & \hat{e}_{3}
\end{array}\right]
$$

To determine principal strain directions of $[B]$, one needs to determine the eigenvalues $\lambda$ of $[U]$ by solving

$$
|[U]-\lambda[I]|=0 .
$$

Equation (36) indicates that the eigenvalues $\lambda_{i}$ will vary with $z$. However, because the membrane thickness $h$ is usually small, one can choose the reference point $(z=0)$ for computing principal strain directions. The eigenvalues of $[U]_{z=0}$ can be obtained to be

$$
\begin{aligned}
\lambda_{1}, \lambda_{2} & =\frac{\hat{e}_{1} \cos \gamma_{61}+\hat{e}_{2} \cos \gamma_{62} \pm \sqrt{\left(\hat{e}_{1} \cos \gamma_{61}+\hat{e}_{2} \cos \gamma_{62}\right)^{2}-4 \hat{e}_{1} \hat{e}_{2} \cos \gamma_{6}}}{2} \\
\lambda_{3} & =1+e_{3}
\end{aligned}
$$

The corresponding eigenvectors are:

$$
\left\{C_{1}\right\}=\left\{C_{11}, C_{21}, 0\right\}^{T}, \quad\left\{C_{2}\right\}=\left\{-C_{21}, C_{11}, 0\right\}^{T}, \quad\left\{C_{3}\right\}=\{0,0,1\}^{T},
$$

where $C_{11}$ and $C_{21}$ can be obtained from Eq. (36) (with $z=0$ ) as functions of $e_{1}$, $e_{2}, \gamma_{61}$, and $\gamma_{62}$. If $\gamma_{61}=\gamma_{62}=0$, eigenvalues of $[U]_{z=0}$ are $1+e_{1}, 1+e_{2}$, and $1+e_{3}$, and $C_{11}=1$ and $C_{21}=0$. In other words, the axes $\xi$, $\eta$, and $\zeta$ are the principal strain axes. Pai and Palazotto ${ }^{13}$ showed that, if $\left\{C_{i}\right\}$ are normalized such that

$$
[C]^{\boldsymbol{T}}[C]=[I],
$$


then

$$
\left\{\mathbf{i}_{\overline{1} \tilde{2} \tilde{3}}\right\}=[C]^{T}\left\{\mathbf{i}_{123}\right\}, \quad[U]=[C]\left[\begin{array}{ccc}
\lambda_{1} & 0 & 0 \\
0 & \lambda_{2} & 0 \\
0 & 0 & \lambda_{3}
\end{array}\right][C]^{T}
$$

where $[C] \equiv\left[\left\{C_{1}\right\}\left\{C_{2}\right\}\left\{C_{3}\right\}\right],\left\{\mathbf{i}_{\overline{1} \check{2} \check{3}}\right\}=\left\{\mathbf{i}_{\mathfrak{1}} \mathbf{i}_{\check{2}} \mathbf{i}_{\overline{3}}\right\}^{T}$, and $\mathbf{i}_{\bar{k}}$ are base vectors along the principal strain directions.

Equations (11b) and (36) show that $[U]_{z=0}$ is symmetric. For strains at $z \neq 0$, $[U]$ is symmetric only if $k_{61}=k_{62}$ and $e_{3 x}=e_{3 y}=0 . k_{61}=k_{62}$ is true if the deformed reference surface is smooth. However, $e_{3 x}$ and $e_{3 y}$ may not be zero and approximations due to computation may make $k_{61} \neq k_{62}$, and hence the $[U]$ in Eq. (36) is usually asymmetric. The transformation matrix $[T]$ in Eq. (14a) is derived only for points on the reference surface (i.e., $z=0$ ), but it is used in Eq. (36) for all points. Hence, the $[U]$ in Eq. (36) is not really the right stretch tensor for points at $z \neq 0$ because of the nonuniform distribution of $e_{3}$ and the local rotations due to twistings (i.e., $\hat{z} k_{61}, \hat{z} k_{62}$ ). Consequently, instead of using Eq. (35), Jaumann strains $B_{m n}$ are modified as:

$$
[B]=\frac{1}{2}\left[[U]+[U]^{T}-[\breve{Y}]-[\breve{Y}]^{T}\right] .
$$

Substituting Eqs. (32) and (36) into Eq. (41) yields the Jaumann strains of a doublycurved membrane as:

$$
\begin{aligned}
& B_{11}=\left(1+e_{1}\right) \cos \gamma_{61}-1+\hat{z} k_{1}-z k_{1}^{0}, \quad B_{22}=\left(1+e_{2}\right) \cos \gamma_{62}-1+\hat{z} k_{2}-z k_{2}^{0}, \\
& B_{12}=\frac{1}{2}\left[\left(1+e_{1}\right) \sin \gamma_{61}+\left(1+e_{2}\right) \sin \gamma_{62}+\hat{z} k_{6}-z k_{6}^{0}\right] \\
& B_{33}=e_{3}, \quad B_{13}=\frac{1}{2} e_{3 x} z, \quad B_{23}=\frac{1}{2} e_{3 y} z
\end{aligned}
$$

where $k_{6} \equiv k_{61}+k_{62}$ and $k_{6}^{0} \equiv k_{61}^{0}+k_{62}^{0}$. As membranes usually have thin thickness, the $z$ is small. Hence, we replace $\hat{z}$ with $z$, neglect $e_{3 x} z$ and $e_{3 y} z$, and rewrite Eq. (42) as:

$$
\{B\}=[S]\{\psi\}, \quad B_{33}=e_{3},
$$

where

$$
\begin{aligned}
\{B\} \equiv & \left\{\begin{array}{c}
B_{11} \\
B_{22} \\
2 B_{12}
\end{array}\right\}, \quad[S] \equiv\left[\begin{array}{cccccc}
1 & 0 & 0 & z & 0 & 0 \\
0 & 1 & 0 & 0 & z & 0 \\
0 & 0 & 1 & 0 & 0 & z
\end{array}\right] \\
\{\psi\} \equiv & \left\{\left(1+e_{1}\right) \cos \gamma_{61}-1,\left(1+e_{2}\right) \cos \gamma_{62}-1,\left(1+e_{1}\right) \sin \gamma_{61}\right. \\
& \left.+\left(1+e_{2}\right) \sin \gamma_{62}, k_{1}-k_{1}^{0}, k_{2}-k_{2}^{0}, k_{6}-k_{6}^{0}\right\}^{T}
\end{aligned}
$$




\subsubsection{Concept of local relative displacements}

Next we show that Jaumann strains can be easily derived using the concept of local relative displacements. ${ }^{14}$ The local displacement vector $u$, of an arbitrary point on a differential membrane element, has the form

$$
\mathbf{u}=\boldsymbol{u}_{\boldsymbol{k}} \mathbf{i}_{\boldsymbol{k}},
$$

where

$$
\begin{aligned}
& u_{1}(x, y, z, t)=u_{1}^{0}(x, y, t)+z\left[\theta_{2}(x, y, t)-\theta_{2}^{0}(x, y)\right], \\
& u_{2}(x, y, z, t)=u_{2}^{0}(x, y, t)-z\left[\theta_{1}(x, y, t)-\theta_{1}^{0}(x, y)\right], \\
& u_{3}(x, y, z, t)=u_{3}^{0}(x, y, t)+z e_{3} .
\end{aligned}
$$

Here, $u_{k}^{0}$ are the displacements (with respect to the system $\xi \eta \zeta$ ) of the reference point $A^{\prime}$ shown in Fig. 1, $\theta_{1}$ and $\theta_{2}$ are the rotation angles of the observed membrane element with respect to the $\xi$ and $\eta$ axes, respectively, and $\theta_{1}^{0}$ and $\theta_{2}^{0}$ are the corresponding initial rotation angles. Because the $\xi \eta \zeta$ is a local coordinate system attached to the observed membrane element, and the $\xi-\eta$ plane is tangent to the deformed reference surface, we have

$$
u_{1}^{0}=u_{2}^{0}=u_{3}^{0}=\theta_{1}^{0}=\theta_{2}^{0}=\theta_{1}=\theta_{2}=\frac{\partial u_{3}^{0}}{\partial x}=\frac{\partial u_{3}^{0}}{\partial y}=0
$$

It follows from Fig. 1 and Eqs. (3), (16), and (17) that

$$
\begin{gathered}
\frac{\partial u_{2}^{0}}{\left(1+e_{1}\right) \partial x}=\sin \gamma_{61}, \quad \frac{\partial u_{1}^{0}}{\left(1+e_{2}\right) \partial y}=\sin \gamma_{62}, \\
\frac{\partial u_{1}^{0}}{\partial x}=\left(1+e_{1}\right) \cos \gamma_{61}-1, \quad \frac{\partial u_{2}^{0}}{\partial y}=\left(1+e_{2}\right) \cos \gamma_{62}-1, \\
\frac{\partial \theta_{2}^{0}}{\partial x}=-\frac{\partial \mathbf{j}_{1}}{\partial x} \cdot \mathbf{j}_{3}=k_{1}^{0}, \quad \frac{\partial \theta_{1}^{0}}{\partial y}=-k_{2}^{0}, \quad \frac{\partial \theta_{1}^{0}}{\partial x}=-k_{61}^{0}, \quad \frac{\partial \theta_{2}^{0}}{\partial y}=k_{62}^{0}, \\
\frac{\partial \theta_{2}}{\partial x}=-\frac{\partial \mathbf{i}_{1}}{\partial x} \cdot \mathbf{i}_{3}=k_{1}, \quad \frac{\partial \theta_{1}}{\partial y}=-k_{2}, \quad \frac{\partial \theta_{1}}{\partial x}=-k_{61}, \quad \frac{\partial \theta_{2}}{\partial y}=k_{62} .
\end{gathered}
$$

Taking the derivatives of Eqs. (45) and (46) and using Eqs. (47) and (48), we obtain

$$
\begin{aligned}
\frac{\partial \mathbf{u}}{\partial x}= & \frac{\partial u_{1}}{\partial x} \mathbf{i}_{1}+\frac{\partial u_{2}}{\partial x} \mathbf{i}_{2}+\frac{\partial u_{3}}{\partial x} \mathbf{i}_{3}+u_{1} \frac{\partial \mathbf{i}_{1}}{\partial x}+u_{2} \frac{\partial \mathbf{i}_{2}}{\partial x}+u_{3} \frac{\partial \mathbf{i}_{3}}{\partial x} \\
= & {\left[\left(1+e_{1}\right) \cos \gamma_{61}-1+\hat{z} k_{1}-z k_{1}^{0}\right] \mathbf{i}_{1}+\left[\left(1+e_{1}\right) \sin \gamma_{61}+\hat{z} k_{61}-z k_{61}^{0}\right] \mathbf{i}_{2} } \\
& +z e_{3 x} \mathbf{i}_{3}, \\
\frac{\partial \mathbf{u}}{\partial y}= & {\left[\left(1+e_{2}\right) \sin \gamma_{62}+\hat{z} k_{62}-z k_{62}^{0}\right] \mathbf{i}_{1}+\left[\left(1+e_{2}\right) \cos \gamma_{62}-1+\hat{z} k_{2}-z k_{2}^{0}\right] \mathbf{i}_{2} } \\
& +z e_{3 y} \mathbf{i}_{3},
\end{aligned}
$$

$$
\frac{\partial \mathbf{u}}{\partial z}=e_{3} \mathbf{i}_{3}
$$


In terms of the local relative displacements shown in Eqs. (45) and (46), Jaumann strains can be simply defined as ${ }^{14}$ :

$$
B_{m n}=\frac{1}{2}\left(\frac{\partial \mathbf{u}}{\partial x_{m}} \cdot \mathbf{i}_{n}+\frac{\partial \mathbf{u}}{\partial x_{n}} \cdot \mathbf{i}_{m}\right), \quad m, n=1,2,3 .
$$

Substituting Eqs. (49)-(51) into Eq. (52) yields the same Jaumann strains shown in Eq. (42).

\subsubsection{Constitutive equation}

For isotropic materials the constitutive equation that relates Jaumann stresses $J_{m n}$ to Jaumann strains $B_{m n}$ is given by:

$$
\left\{\begin{array}{l}
J_{11} \\
J_{22} \\
J_{33} \\
J_{12}
\end{array}\right\}=\frac{E}{(1+\nu)(1-2 \nu)}\left[\begin{array}{cccc}
1-\nu & \nu & \nu & 0 \\
\nu & 1-\nu & \nu & 0 \\
\nu & \nu & 1-\nu & 0 \\
0 & 0 & 0 & \frac{1-2 \nu}{2}
\end{array}\right]\left\{\begin{array}{c}
B_{11} \\
B_{22} \\
B_{33} \\
2 B_{12}
\end{array}\right\},
$$

where $E$ is Young's modulus and $\nu$ is Poisson's ratio. We note that, for a material with $\nu=0.5$ (e.g., rubber), numerical singularity exists in the constitutive equation. However, because the thickness of a membrane is usually thin and hence $B_{33}\left(=e_{3}\right)$ is mainly due to Poisson's effect, one can assume that $J_{33}=0$ to obtain that

$$
e_{3}=\frac{\nu}{\nu-1} B_{11}+\frac{\nu}{\nu-1} B_{22}
$$

Consequently, we have

$$
\left\{\begin{array}{l}
J_{11} \\
J_{22} \\
J_{12}
\end{array}\right\}=\frac{E}{1-\nu^{2}}\left[\begin{array}{ccc}
1 & \nu & 0 \\
\nu & 1 & 0 \\
0 & 0 & \frac{1-\nu}{2}
\end{array}\right]\left\{\begin{array}{c}
B_{11} \\
B_{22} \\
2 B_{12}
\end{array}\right\} .
$$

Membrane materials can be anisotropic. For example, Mylar has been reported to be orthotropic. ${ }^{11}$ If the membrane is made of layers of orthotropic materials, one can obtain the transformed stiffness matrix $\left[\bar{Q}^{(i)}\right]$ for the $i$ th lamina from its principal stiffness matrix $\left[Q^{(i)}\right]$ and its ply angle (measured with respect to the axis $x$ ) by using tensor transformation and assuming $J_{33}^{(i)}=0$. Then, the constitutive equation for the $i$ th lamina is given by:

$$
\{J\}=\left[\bar{Q}^{(i)}\right]\{B\},
$$

where

$$
\{J\} \equiv\left\{\begin{array}{c}
J_{11}^{(i)} \\
J_{22}^{(i)} \\
J_{12}^{(i)}
\end{array}\right\}, \quad\left[\bar{Q}^{(i)}\right] \equiv\left[\begin{array}{lll}
\bar{Q}_{11}^{(i)} & \bar{Q}_{12}^{(i)} & \bar{Q}_{16}^{(i)} \\
\bar{Q}_{12}^{(i)} & \bar{Q}_{22}^{(i)} & \bar{Q}_{26}^{(i)} \\
\bar{Q}_{16}^{(i)} & \bar{Q}_{26}^{(i)} & \bar{Q}_{66}^{(i)}
\end{array}\right]
$$


Moreover, stress resultants are defined as:

$$
\begin{aligned}
\left\{\begin{array}{l}
N_{1} \\
N_{2} \\
N_{6} \\
M_{1} \\
M_{2} \\
M_{6}
\end{array}\right\} \equiv \int_{z}\left\{\begin{array}{c}
J_{11}^{(i)} \\
J_{22}^{(i)} \\
J_{12}^{(i)} \\
z J_{11}^{(i)} \\
z J_{22}^{(i)} \\
z J_{12}^{(i)}
\end{array}\right\} d z=[\tilde{Q}]\{\psi\}, \\
{[\tilde{Q}] \equiv \int_{z}\left[\begin{array}{c}
{\left[\bar{Q}^{(i)}\right][S]} \\
z\left[\bar{Q}^{(i)}\right][S]
\end{array}\right] d z=\int_{z}\left[\begin{array}{ll}
{\left[\bar{Q}^{(i)}\right]} & z\left[\bar{Q}^{(i)}\right] \\
z\left[\bar{Q}^{(i)}\right] & z^{2}\left[\bar{Q}^{(i)}\right]
\end{array}\right] d z, }
\end{aligned}
$$

where Eqs. (43) and (44) were used and $[\tilde{Q}]$ is a $6 \times 6$ matrix. Here $M_{1}, M_{2}$, and $M_{6}$ represent the moment intensities acting on the edges of a differential membrane element, $N_{1}$ and $N_{2}$ are the in-plane extension force intensities, and $N_{6}$ is the in-plane shear force intensity.

\subsection{Governing equations}

To derive governing equations we use the extended Hamilton's principle, which states that $^{15}$

$$
\int_{0}^{t}\left(\delta T-\delta \Pi+\delta W_{n c}\right) d t=0,
$$

where $t$ is the time, $\delta T$ is the variation of kinetic energy, $\delta \Pi$ is the variation of elastic energy, and $\delta W_{n c}$ is the variation of nonconservative work.

Deformations of membranes are mainly caused by rigid-body displacements and rotations and in-plane stretching, and the in-plane shear strain $\gamma_{6}\left(=\gamma_{61}+\gamma_{62}\right)$ is usually small. Hence, the influence of $\gamma_{6}$ on the deformed geometry can be neglected without significant loss of accuracy in accounting for geometric nonlinearities. Substituting $\gamma_{61}=\gamma_{62}=\gamma_{6}=0$ into Eqs. (14a) and (14b) yields

$$
[\Gamma]=[I], \quad \hat{T}_{1 i}=T_{1 i}, \quad \hat{T}_{2 i}=T_{2 i}, \quad \mathbf{i}_{1}=\mathbf{i}_{\hat{1}}, \quad \mathbf{i}_{2}=\mathbf{i}_{\hat{2}} .
$$

Substituting $\gamma_{61}=\gamma_{62}=\gamma_{6}=0$ and Eq. (58) into Eqs. (19), (20), (27), and (28) yields

$$
\begin{aligned}
\delta e_{1}= & T_{11} \delta\left(u_{x}-v k_{5}^{0}+w k_{1}^{0}\right)+T_{12} \delta\left(v_{x}+u k_{5}^{0}+w k_{61}^{0}\right) \\
& +T_{13} \delta\left(w_{x}-u k_{1}^{0}-v k_{61}^{0}\right) \\
\delta e_{2}= & T_{21} \delta\left(u_{y}-v k_{4}^{0}+w k_{62}^{0}\right)+T_{22} \delta\left(v_{y}+u k_{4}^{0}+w k_{2}^{0}\right) \\
& +T_{23} \delta\left(w_{y}-u k_{62}^{0}-v k_{2}^{0}\right)
\end{aligned}
$$




$$
\begin{aligned}
& -\left(1+e_{2}\right) \delta \theta_{1}+T_{31}\left(\delta u_{y}-k_{4}^{0} \delta v+k_{62}^{0} \delta w\right)+T_{32}\left(\delta v_{y}+k_{4}^{0} \delta u+k_{2}^{0} \delta w\right) \\
& \quad+T_{33}\left(\delta w_{y}-k_{62}^{0} \delta u-k_{2}^{0} \delta v\right)=0 \\
& \left(1+e_{1}\right) \delta \theta_{2}+T_{31}\left(\delta u_{x}-k_{5}^{0} \delta v+k_{1}^{0} \delta w\right)+T_{32}\left(\delta v_{x}+k_{5}^{0} \delta u+k_{61}^{0} \delta w\right) \\
& \quad+T_{33}\left(\delta w_{x}-k_{1}^{0} \delta u-k_{61}^{0} \delta v\right)=0
\end{aligned}
$$

Using Eq. (58) and taking the variations of $\mathbf{i}_{1}\left(=\mathbf{i}_{\hat{1}}\right)$ and $\mathbf{i}_{2}\left(=\mathbf{i}_{\hat{2}}\right)$ in Eqs. (8) and (9), we obtain

$$
\begin{aligned}
\delta \mathbf{i}_{1}= & \mathbf{j}_{1} \delta T_{11}+\mathbf{j}_{2} \delta T_{12}+\mathbf{j}_{3} \delta T_{13} \\
= & {\left[\left(\delta u_{x}-k_{5}^{0} \delta v+k_{1}^{0} \delta w\right) \mathbf{j}_{1}+\left(\delta v_{x}+k_{5}^{0} \delta u+k_{61}^{0} \delta w\right) \mathbf{j}_{2}+\left(\delta w_{x}-k_{1}^{0} \delta u-k_{61}^{0} \delta v\right) \mathbf{j}_{3}\right] } \\
& \times \frac{1}{1+e_{1}}-\frac{\delta e_{1}}{1+e_{1}} \mathbf{i}_{1}, \\
\delta \mathbf{i}_{2}= & \mathbf{j}_{1} \delta T_{21}+\mathbf{j}_{2} \delta T_{22}+\mathbf{j}_{3} \delta T_{23} \\
= & {\left[\left(\delta u_{y}-k_{4}^{0} \delta v+k_{62}^{0} \delta w\right) \mathbf{j}_{1}+\left(\delta v_{y}+k_{4}^{0} \delta u+k_{2}^{0} \delta w\right) \mathbf{j}_{2}+\left(\delta w_{y}-k_{62}^{0} \delta u-k_{2}^{0} \delta v\right) \mathbf{j}_{3}\right] } \\
& \times \frac{1}{1+e_{2}}-\frac{\delta e_{2}}{1+e_{2}} \mathbf{i}_{2} .
\end{aligned}
$$

Using Eqs. (61a) and (61b) and considering $\gamma_{61}$ and $\gamma_{62}$ being small, we obtain that

$$
\begin{aligned}
\delta \gamma_{61}= & \delta \mathbf{i}_{\hat{1}} \cdot \mathbf{i}_{2} \\
= & \frac{1}{1+e_{1}}\left[T_{21}\left(\delta u_{x}-k_{5}^{0} \delta v+k_{1}^{0} \delta w\right)+T_{22}\left(\delta v_{x}+k_{5}^{0} \delta u+k_{61}^{0} \delta w\right)\right. \\
& \left.+T_{23}\left(\delta w_{x}-k_{1}^{0} \delta u-k_{61}^{0} \delta v\right)\right], \\
\delta \gamma_{62}= & \delta \mathbf{i}_{\hat{2}} \cdot \mathbf{i}_{1} \\
= & \frac{1}{1+e_{2}}\left[T_{11}\left(\delta u_{y}-k_{4}^{0} \delta v+k_{62}^{0} \delta w\right)+T_{12}\left(\delta v_{y}+k_{4}^{0} \delta u+k_{2}^{0} \delta w\right)\right. \\
& \left.+T_{13}\left(\delta w_{y}-k_{62}^{0} \delta u-k_{2}^{0} \delta v\right)\right] .
\end{aligned}
$$

Moreover, when $\gamma_{6}, \gamma_{61}$ and $\gamma_{62}$ are small, the Jaumann strains in Eq. (44) reduce to

$$
\begin{gathered}
B_{11}=e_{1}+z\left(k_{1}-k_{1}^{0}\right), \quad B_{22}=e_{2}+z\left(k_{2}-k_{2}^{0}\right), \\
2 B_{12}=\gamma_{61}+\gamma_{62}+z\left(k_{6}-k_{6}^{0}\right) .
\end{gathered}
$$

It follows from Eqs. (30a) and (30b) that the displacement vector D of an arbitrary point on the differential membrane element is:

$$
\mathrm{D}=\mathbf{P}_{q}-\mathbf{P}_{Q}=u \mathbf{j}_{1}+v \mathbf{j}_{2}+w \mathbf{j}_{3}+\hat{z} \mathbf{i}_{3}-z \mathbf{j}_{3}
$$


Taking the variation of Eq. (64) and using Eq. (25) yields

$$
\delta \mathbf{D}=\mathbf{j}_{1} \delta u+\mathbf{j}_{2} \delta v+\mathbf{j}_{3} \delta w+\hat{z}\left(\mathbf{i}_{1} \delta \theta_{2}-\mathbf{i}_{2} \delta \theta_{1}\right)
$$

Taking time derivatives of $\mathbf{D}$ and using Eq. (14a) yields

$$
\ddot{\mathbf{D}}=\ddot{u} \mathbf{j}_{1}+\ddot{v} \mathbf{j}_{2}+\ddot{w} \mathbf{j}_{3}+\hat{z}\left(\ddot{T}_{31} \mathbf{j}_{1}+\ddot{T}_{32} \mathbf{j}_{2}+\ddot{T}_{33} \mathbf{j}_{3}\right) \text {. }
$$

Using Eqs. (65a), (65b), and (14a), we obtain the variation of kinetic energy as:

$$
\begin{aligned}
\delta T & =-\int_{z} \int_{A} \rho \ddot{\mathbf{D}} \cdot \delta \mathbf{D} d x d y d z \\
& =-\int_{A}\left(A_{u} \delta u+A_{v} \delta v+A_{w} \delta w+A_{\theta_{1}} \delta \theta_{1}+A_{\theta_{2}} \delta \theta_{2}\right) d x d y,
\end{aligned}
$$

where $A$ is the area of the undeformed reference surface, $\rho$ is the mass density per unit volume, and

$$
\begin{aligned}
A_{u} & =m \ddot{u}+I_{1} \ddot{T}_{31}, \quad A_{v}=m \ddot{v}+I_{1} \ddot{T}_{32}, \quad A_{w}=m \ddot{w}+I_{1} \ddot{T}_{33}, \\
A_{\theta_{1}} & =-I_{1}\left(\ddot{u} T_{21}+\ddot{v} T_{22}+\ddot{w} T_{23}\right)-I_{2}\left(\ddot{T}_{31} T_{21}+\ddot{T}_{32} T_{22}+\ddot{T}_{33} T_{23}\right), \\
A_{\theta_{2}} & =I_{1}\left(\ddot{u} T_{11}+\ddot{v} T_{12}+\ddot{w} T_{13}\right)+I_{2}\left(\ddot{T}_{31} T_{11}+\ddot{T}_{32} T_{12}+\ddot{T}_{33} T_{13}\right), \\
\left\{m, I_{1}, I_{2}\right\} & \equiv \int_{z} \rho\left\{1, \hat{z}, \hat{z}^{2}\right\} d z,
\end{aligned}
$$

where $m$ represents the mass per unit area and $\hat{z}=\left(1+e_{3}\right) z$. If $\rho$ is symmetric with respect to the middle reference surface, $I_{1}=0$. Moreover, we also assume that the membrane thickness $h$ is small and hence the rotary inertia density $I_{2}$ is negligible. Substituting $I_{1}=I_{2}=0$ into Eq. (66) yields

$$
\delta T=-\int_{A}(m \ddot{u} \delta u+m \ddot{v} \delta v+m \ddot{w} \delta w) d x d y .
$$

If the membrane is only subjected to a normal pressure $p$ along the $\zeta$ axis, the nonconservative work is given by:

$$
\begin{aligned}
\delta W_{n c} & =\int_{A} p\left(1+e_{1}\right) d x\left(1+e_{2}\right) d y \mathbf{i}_{3} \cdot \delta\left(u \mathbf{j}_{1}+v \mathbf{j}_{2}+w \mathbf{j}_{3}\right) \\
& =\int_{A} p\left(T_{31} \delta u+T_{32} \delta v+T_{33} \delta w\right)\left(1+e_{1}\right)\left(1+e_{2}\right) d x d y \\
& =\int_{A}\left(p_{1} \delta u+p_{2} \delta v+p_{3} \delta w\right) d x d y,
\end{aligned}
$$

where Eq. (14a) is used and the actual deformed area $\left(1+e_{1}\right) d x\left(1+e_{2}\right) d y$ is used to calculate the actual load. Moreover,

$$
\begin{gathered}
p_{1} \equiv p\left(1+e_{1}\right)\left(1+e_{2}\right) T_{31}, \quad p_{2} \equiv p\left(1+e_{1}\right)\left(1+e_{2}\right) T_{32}, \\
p_{3} \equiv p\left(1+e_{1}\right)\left(1+e_{2}\right) T_{33} .
\end{gathered}
$$


Because the elastic energy $\Pi$ is due to relative displacements among material particles, $\delta \Pi$ is the virtual work done by the internal forces $\mathbf{f}_{i}$ through the virtual relative displacement $\delta \mathrm{u}$. Hence, we have

$$
\begin{aligned}
\delta \Pi= & \int_{V^{0}}\left[\mathbf{f}_{1} \cdot \mathbf{i}_{n} \delta\left(\frac{\partial \mathbf{u}}{\partial x_{1}} d x_{1} \cdot \mathbf{i}_{n}\right)+\mathbf{f}_{2} \cdot \mathbf{i}_{n} \delta\left(\frac{\partial \mathbf{u}}{\partial x_{2}} d x_{2} \cdot \mathbf{i}_{n}\right)\right. \\
& \left.+\mathbf{f}_{3} \cdot \mathbf{i}_{n} \delta\left(\frac{\partial \mathbf{u}}{\partial x_{3}} d x_{3} \cdot \mathbf{i}_{n}\right)\right]
\end{aligned}
$$

where $d x_{1} \equiv d x, d x_{2} \equiv d y, d x_{3} \equiv d z, d V^{0}=d x d y d z$, and $\mathbf{f}_{i}$ is the force vector acting on the convected area of $d x_{m} d x_{n}(i \neq m \neq n)$. Because Jaumann stresses are defined as ${ }^{13}$ :

$$
J_{11}=\frac{\mathbf{f}_{1} \cdot \mathbf{i}_{1}}{d x_{2} d x_{3}}, \quad J_{22}=\frac{\mathbf{f}_{2} \cdot \mathbf{i}_{2}}{d x_{1} d x_{3}}, \quad J_{12}=\frac{\mathbf{f}_{1} \cdot \mathbf{i}_{2}}{d x_{2} d x_{3}}=\frac{\mathbf{f}_{2} \cdot \mathbf{i}_{1}}{d x_{1} d x_{3}}=J_{21},
$$

substituting Eqs. (71b) and (52) into Eq. (71a) and using $B_{13}=B_{23}=J_{33}=0$ yields

$$
\delta \Pi=\int_{A} \int_{z}\left(J_{11} \delta B_{11}+J_{22} \delta B_{22}+2 J_{12} \delta B_{12}\right) d x d y d z
$$

Substituting Eq. (63) into Eq. (72), using Eqs. (26a) and (26b), and integrating by parts we obtain that

$$
\begin{aligned}
\delta \Pi= & \int_{A} \int_{z}\left[J_{11}\left(\delta e_{1}+z \delta k_{1}\right)+J_{22}\left(\delta e_{2}+z \delta k_{2}\right)+J_{12}\left(\delta \gamma_{61}+\delta \gamma_{62}+z \delta k_{6}\right)\right] d x d y d z \\
= & \int_{A}\left(N_{1} \delta e_{1}+N_{2} \delta e_{2}+N_{6} \delta \gamma_{61}+N_{6} \delta \gamma_{62}+M_{1} \delta k_{1}+M_{2} \delta k_{2}+M_{6} \delta k_{6}\right) d x d y \\
= & \int_{A}\left(N_{1} \delta e_{1}+N_{2} \delta e_{2}+N_{6} \delta \gamma_{61}+N_{6} \delta \gamma_{62}+\Theta_{1} \delta \theta_{1}+\Theta_{2} \delta \theta_{2}+\Theta_{3} \delta \theta_{3}\right) d x d y \\
& +\int_{x}\left[-M_{2} \delta \theta_{1}+M_{6} \delta \theta_{2}\right]_{y=0}^{y=Y} d x+\int_{y}\left[-M_{6} \delta \theta_{1}+M_{1} \delta \theta_{2}\right]_{x=0}^{x=X} d y,
\end{aligned}
$$

where $X$ and $Y$ denote the integration limits of the curvilinear coordinates $x$ and $y$, respectively, and

$$
\begin{aligned}
& \Theta_{1} \equiv M_{6 x}+M_{2 y}+M_{1} k_{5}+M_{6} k_{4} \\
& \Theta_{2} \equiv-M_{1 x}-M_{6 y}+M_{2} k_{4}+M_{6} k_{5} \\
& \Theta_{3} \equiv M_{1} k_{61}-M_{2} k_{62}+M_{6} k_{2}-M_{6} k_{1} .
\end{aligned}
$$


Moreover, it follows from Eqs. (63), (44) and (56) that

$$
\left\{\begin{array}{l}
N_{1} \\
N_{2} \\
N_{6} \\
M_{1} \\
M_{2} \\
M_{6}
\end{array}\right\}=[\tilde{Q}]\left\{\begin{array}{c}
e_{1} \\
e_{2} \\
\gamma_{6} \\
k_{1}-k_{1}^{0} \\
k_{2}-k_{2}^{0} \\
k_{6}-k_{6}^{0}
\end{array}\right\} .
$$

Substituting Eqs. (59a), (59b), (62a), and (62b) into Eq. (73) and adding the integrals of $Q_{2} \times$ Eq. (60a) and $Q_{1} \times$ Eq. (60b), we obtain

$$
\begin{aligned}
& \delta \Pi=\int_{A}\left\{\left\{N_{1}, \frac{N_{6}}{1+e_{1}}, Q_{1}\right\}[T]\{\delta u v w\}_{x}+\left\{N_{1}, \frac{N_{6}}{1+e_{1}}, Q_{1}\right\}[T]\left[K_{1}^{0}\right]^{T}\{\delta u v w\}\right. \\
& +\left\{\frac{N_{6}}{1+e_{2}}, N_{2}, Q_{2}\right\}[T]\{\delta u v w\}_{y}+\left\{\frac{N_{6}}{1+e_{2}}, N_{2}, Q_{2}\right\}[T]\left[K_{2}^{0}\right]^{T}\{\delta u v w\} \\
& \left.+\left[\Theta_{1}-Q_{2}\left(1+e_{2}\right)\right] \delta \theta_{1}+\left[\Theta_{2}+Q_{1}\left(1+e_{1}\right)\right] \delta \theta_{2}+\Theta_{3} \delta \theta_{3}\right\} d x d y \\
& +\int_{x}\left[-M_{2} \delta \theta_{1}+M_{6} \delta \theta_{2}\right]_{y=0}^{y=Y} d x+\int_{y}\left[-M_{6} \delta \theta_{1}+M_{1} \delta \theta_{2}\right]_{x=0}^{x=X} d y
\end{aligned}
$$

where $\{u v w\} \equiv\left\{\begin{array}{lll}u & v & w\end{array}\right\}^{T}$. It is shown later that the introduced $Q_{1}$ and $Q_{2}$ are transverse shear force intensities.

Substituting Eqs. (68), (69), (75), and (74a) into Eq. (57), integrating by parts, and using Eqs. (60a) and (60b) for the boundary terms $-M_{6} \delta \theta_{1}$ and $M_{6} \delta \theta_{2}$ in Eq. (75), we obtain

$$
\begin{aligned}
& \int_{A}\left\{\left(\frac{\partial\left\{F_{\alpha}\right\}^{T}[T]}{\partial x}-\left\{F_{\alpha}\right\}^{T}[T]\left[K_{1}^{0}\right]^{T}+\frac{\partial\left\{F_{\beta}\right\}^{T}[T]}{\partial y}-\left\{F_{\beta}\right\}^{T}[T]\left[K_{2}^{0}\right]^{T}\right)\{\delta u v w\}\right. \\
& +\left(\left\{R_{F}\right\}^{T}-\left\{I_{F}\right\}^{T}\right)\{\delta u v w\}+\left(\left\{Q_{\alpha}\right\}^{T}+\left\{Q_{\beta}\right\}^{T}\right)\{\delta \theta\} \\
& \left.+\left(\frac{\partial\left\{M_{\alpha}\right\}^{T}}{\partial x}+\left\{M_{\alpha}\right\}^{T}\left[K_{1}\right]+\frac{\partial\left\{M_{\beta}\right\}^{T}}{\partial y}+\left\{M_{\beta}\right\}^{T}\left[K_{2}\right]\right)\{\delta \theta\}\right\} d x d y \\
& -\int_{x}\left[\left\{F_{\beta}\right\}^{T}[T]\{\delta u v w\}+\left[\left(\frac{M_{6} T_{31}}{1+e_{1}}\right)_{x}+\frac{M_{6}}{1+e_{1}}\left(T_{33} k_{1}^{0}-T_{32} k_{5}^{0}\right)\right] \delta u\right. \\
& \quad+\left[\left(\frac{M_{6} T_{32}}{1+e_{1}}\right)_{x}+\frac{M_{6}}{1+e_{1}}\left(T_{33} k_{61}^{0}+T_{31} k_{5}^{0}\right)\right] \delta v \\
& \left.\quad+\left[\left(\frac{M_{6} T_{33}}{1+e_{1}}\right)_{x}-\frac{M_{6}}{1+e_{1}}\left(T_{32} k_{61}^{0}+T_{31} k_{1}^{0}\right)\right] \delta w-M_{2} \delta \theta_{1}\right]_{y=0}^{y=Y} d x
\end{aligned}
$$




$$
\begin{aligned}
& -\int_{y}\left[\left\{F_{\alpha}\right\}^{T}[T]\{\delta u v w\}+\left[\left(\frac{M_{6} T_{31}}{1+e_{2}}\right)_{y}+\frac{M_{6}}{1+e_{2}}\left(T_{33} k_{62}^{0}-T_{32} k_{4}^{0}\right)\right] \delta u\right. \\
& +\left[\left(\frac{M_{6} T_{32}}{1+e_{2}}\right)_{y}+\frac{M_{6}}{1+e_{2}}\left(T_{33} k_{2}^{0}+T_{31} k_{4}^{0}\right)\right] \delta v \\
& \left.+\left[\left(\frac{M_{6} T_{33}}{1+e_{2}}\right)_{y}-\frac{M_{6}}{1+e_{2}}\left(T_{32} k_{2}^{0}+T_{31} k_{62}^{0}\right)\right] \delta w+M_{1} \delta \theta_{2}\right]_{x=0}^{x=X} d x \\
& \quad+\left[\left(\frac{M_{6}}{1+e_{1}}+\frac{M_{6}}{1+e_{2}}\right)\left(T_{31} \delta u+T_{32} \delta v+T_{33} \delta w\right)\right]_{(x, y)=(X, 0),(0, Y)}^{(x, y)=(0,0),(X, Y)}=0
\end{aligned}
$$

where

$$
\begin{aligned}
& \{\theta\} \equiv\left\{\theta_{1}, \theta_{2}, \theta_{3}\right\}^{T}, \\
& \left\{I_{F}\right\} \equiv\{m \ddot{u}, m \ddot{v}, m \ddot{w}\}^{T}, \quad\left\{R_{F}\right\} \equiv\left\{p_{1}, p_{2}, p_{3}\right\}^{T}, \\
& \left\{F_{\alpha}\right\} \equiv\left\{N_{1}, \frac{N_{6}}{1+e_{1}}, Q_{1}\right\}^{T}, \quad\left\{F_{\beta}\right\} \equiv\left\{\frac{N_{6}}{1+e_{2}}, N_{2}, Q_{2}\right\}^{T} \text {, } \\
& \left\{M_{\alpha}\right\} \equiv\left\{-M_{6}, M_{1}, 0\right\}^{T}, \quad\left\{M_{\beta}\right\} \equiv\left\{-M_{2}, M_{6}, 0\right\}^{T}, \\
& \left\{Q_{\alpha}\right\} \equiv\left\{0,-Q_{1}\left(1+e_{1}\right), N_{6}\right\}^{T}, \quad\left\{Q_{\beta}\right\} \equiv\left\{Q_{2}\left(1+e_{2}\right), 0,-N_{6}\right\}^{T} .
\end{aligned}
$$

Setting the coefficients of $\delta u, \delta v, \delta w, \delta \theta_{1}, \delta \theta_{2}$, and $\delta \theta_{3}$ in Eq. (76) equal to zero, we obtain the equations of motion as:

$$
\begin{gathered}
\frac{\partial}{\partial x}\left([T]^{T}\left\{F_{\alpha}\right\}\right)-\left[K_{1}^{0}\right][T]^{T}\left\{F_{\alpha}\right\}+\frac{\partial}{\partial y}\left([T]^{T}\left\{F_{\beta}\right\}\right)-\left[K_{2}^{0}\right][T]^{T}\left\{F_{\beta}\right\}+\left\{R_{F}\right\}=\left\{I_{F}\right\} \\
\frac{\partial\left\{M_{\alpha}\right\}}{\partial x}+\left[K_{1}\right]^{T}\left\{M_{\alpha}\right\}+\frac{\partial\left\{M_{\beta}\right\}}{\partial y}+\left[K_{2}\right]^{T}\left\{M_{\beta}\right\}+\left\{Q_{\alpha}\right\}+\left\{Q_{\beta}\right\}=\{0\}
\end{gathered}
$$

The boundary conditions are to specify:

Along $x=0, X$ :

$$
\begin{array}{lll}
\delta u=0 & \text { or } & \mathbf{F}_{\alpha} \cdot \mathbf{j}_{1}+\left[\frac{M_{6} T_{31}}{1+e_{2}}\right]_{y}+\frac{\left(T_{33} k_{62}^{0}-T_{32} k_{4}^{0}\right) M_{6}}{1+e_{2}} \\
\delta v=0 & \text { or } & \mathbf{F}_{\alpha} \cdot \mathbf{j}_{2}+\left[\frac{M_{6} T_{32}}{1+e_{2}}\right]_{y}+\frac{\left(T_{33} k_{2}^{0}+T_{31} k_{4}^{0}\right) M_{6}}{1+e_{2}} \\
\delta w=0 & \text { or } \quad \mathbf{F}_{\alpha} \cdot \mathbf{j}_{3}+\left[\frac{M_{6} T_{33}}{1+e_{2}}\right]_{y}-\frac{\left(T_{32} k_{2}^{0}+T_{31} k_{62}^{0}\right) M_{6}}{1+e_{2}} \\
\delta \theta_{2}=0 & \text { or } \quad M_{1}
\end{array}
$$


Along $y=0, Y$ :

$$
\begin{gathered}
\delta u=0 \quad \text { or } \quad \mathbf{F}_{\beta} \cdot \mathbf{j}_{1}+\left[\frac{M_{6} T_{31}}{1+e_{1}}\right]_{x}+\frac{\left(T_{33} k_{1}^{0}-T_{32} k_{5}^{0}\right) M_{6}}{1+e_{1}}, \\
\delta v=0 \quad \text { or } \quad \mathbf{F}_{\beta} \cdot \mathbf{j}_{2}+\left[\frac{M_{6} T_{32}}{1+e_{1}}\right]_{x}+\frac{\left(T_{33} k_{61}^{0}+T_{31} k_{5}^{0}\right) M_{6}}{1+e_{1}}, \\
\delta w=0 \quad \text { or } \quad \mathbf{F}_{\beta} \cdot \mathbf{j}_{3}+\left[\frac{M_{6} T_{33}}{1+e_{1}}\right]_{x}-\frac{\left(T_{31} k_{1}^{0}+T_{32} k_{61}^{0}\right) M_{6}}{1+e_{1}}, \\
\delta \theta_{1}=0 \quad \text { or } \quad M_{2} \cdot \\
\text { At }(x, y)=(0,0),(0, Y),(X, 0),(X, Y): \\
\delta u=0 \text { or }\left[\frac{M_{6}}{1+e_{1}}+\frac{M_{6}}{1+e_{2}}\right] T_{31} \\
\delta v=0 \text { or }\left[\frac{M_{6}}{1+e_{1}}+\frac{M_{6}}{1+e_{2}}\right] T_{32} \\
\delta w=0 \text { or }\left[\frac{M_{6}}{1+e_{1}}+\frac{M_{6}}{1+e_{2}}\right] T_{33}
\end{gathered}
$$

where

$$
\begin{aligned}
& \mathbf{F}_{\alpha}=N_{1} \mathbf{i}_{1}+\frac{N_{6}}{1+e_{1}} \mathbf{i}_{2}+Q_{1} \mathbf{i}_{3} \\
& \mathbf{F}_{\beta}=\frac{N_{6}}{1+e_{2}} \mathbf{i}_{1}+N_{2} \mathbf{i}_{2}+Q_{2} \mathbf{i}_{3}
\end{aligned}
$$

We also define

$$
\begin{aligned}
\mathbf{M}_{\alpha} & =-M_{6} \mathbf{i}_{1}+M_{1} \mathbf{i}_{2}, \\
\mathbf{M}_{\beta} & =-M_{2} \mathbf{i}_{1}+M_{6} \mathbf{i}_{2}, \\
\mathbf{I}_{F} & =m \ddot{u} \mathbf{j}_{1}+m \ddot{v} \mathbf{j}_{2}+m \ddot{w} \mathbf{j}_{3}, \\
\mathbf{R}_{F} & =p_{1} \mathbf{j}_{1}+p_{2} \mathbf{j}_{2}+p_{3} \mathbf{j}_{3} .
\end{aligned}
$$

Using Eqs. (16) and (17) and the identity $[T]^{T}=[T]^{-1}$, we rewrite Eq. (78) as:

$$
[T]^{T}\left(\frac{\partial\left\{F_{\alpha}\right\}}{\partial x}+\left[K_{1}\right]^{T}\left\{F_{\alpha}\right\}+\frac{\partial\left\{F_{\beta}\right\}}{\partial y}+\left[K_{2}\right]^{T}\left\{F_{\beta}\right\}\right)+\left\{R_{F}\right\}=\left\{I_{F}\right\}
$$

Using Eqs. (81a), (81b), (16), and (17), we put Eqs. (82) and (79) in the following vector forms:

$$
\begin{gathered}
\frac{\partial \mathbf{F}_{\alpha}}{\partial x}+\frac{\partial \mathbf{F}_{\beta}}{\partial y}+\mathbf{R}_{\boldsymbol{F}}=\mathbf{I}_{\boldsymbol{F}} \\
\frac{\partial \mathbf{M}_{\alpha}}{\partial x}+\frac{\partial \mathbf{M}_{\beta}}{\partial y}+\left(1+e_{1}\right) \mathbf{i}_{1} \times \mathbf{F}_{\alpha}+\left(1+e_{2}\right) \mathbf{i}_{2} \times \mathbf{F}_{\beta}=0
\end{gathered}
$$

Equations (83) and (84) state the balance of forces and moments on a differential membrane element, which can be directly obtained using the stress resultants in Eq. (74b) and a vector approach with Newton's second law. Although we started with the energy formulation (see Eq. (57)), the results are fully correlated with 
the Newtonian formulation and every structural term is interpretable in terms of vectors. This shows that the equations obtained are intrinsic.

One can see from Eq. (81a) that $Q_{1}$ and $Q_{2}$ are along the $\mathbf{i}_{3}$-direction and hence represent the transverse shear force intensities. However, it is assumed that there are no transverse shear strains in this membrane theory and hence $Q_{1}$ and $Q_{2}$ are not directly related to displacement variables (see Eq. (74b)). Hence, $Q_{1}$ and $Q_{2}$ need to be obtained from the first two equations of Eq. (79) as:

$$
\begin{aligned}
Q_{1} & =\frac{1}{1+e_{1}}\left(M_{1 x}+M_{6 y}-M_{2} k_{4}-M_{6} k_{5}\right), \\
Q_{2} & =\frac{1}{1+e_{2}}\left(M_{2 y}+M_{6 x}+M_{6} k_{4}+M_{1} k_{5}\right) .
\end{aligned}
$$

The third equation of Eq. (79) is a statement of the balance of internal moments with respect to the $\zeta$ axis, which is given by:

$$
N_{6}-N_{6}+M_{2} k_{62}-M_{1} k_{61}+M_{6} k_{1}-M_{6} k_{2}=0 .
$$

Hence, only the three equations in Eq. (82) and the boundary conditions in Eq. (80) need to be solved in the nonlinear analysis of doubly-curved membranes. Equation (82) can be rewritten as:

$$
\left\{\begin{array}{c}
N_{1 x}+N_{62 y} \\
N_{61 x}+N_{2 y} \\
Q_{1 x}+Q_{2 y}
\end{array}\right\}+\left\{\begin{array}{c}
k_{1} Q_{1}-k_{5} N_{61}+k_{62} Q_{2}-k_{4} N_{2} \\
k_{61} Q_{1}+k_{5} N_{1}+k_{2} Q_{2}+k_{4} N_{62} \\
-k_{1} N_{1}-k_{61} N_{61}-k_{62} N_{62}-k_{2} N_{2}
\end{array}\right\}+[T]\left\{\begin{array}{c}
p_{1} \\
p_{2} \\
p_{3}
\end{array}\right\}=[T]\left\{\begin{array}{c}
m \ddot{u} \\
m \ddot{v} \\
m \ddot{w}
\end{array}\right\},
$$

where $N_{61} \equiv N_{6} /\left(1+e_{1}\right)$ and $N_{62} \equiv N_{6} /\left(1+e_{2}\right)$.

\subsubsection{Without bending stiffness}

If $\gamma_{6}$ is small and the membrane thickness $h$ is so thin that the change of strain values with $z$ is negligible, the strains in Eq. (63) can be approximated as:

$$
B_{11}=e_{1}, \quad B_{22}=e_{2}, \quad 2 B_{12}=\gamma_{61}+\gamma_{62} .
$$

Substituting Eq. (87) into Eqs. (72) and (73) reveals that $M_{1}, M_{2}$, and $M_{6}$ should disappear from the governing equations. Hence, Eq. (85b) becomes a null statement of $N_{6}=N_{6}$, and Eq. (85a) becomes

$$
Q_{1}=Q_{2}=0 .
$$

Substituting Eq. (88) into Eq. (86) yields

$$
\left\{\begin{array}{c}
N_{1 x}+N_{62 y} \\
N_{61 x}+N_{2 y} \\
0
\end{array}\right\}+\left\{\begin{array}{c}
-k_{5} N_{61}-k_{4} N_{2} \\
k_{5} N_{1}+k_{4} N_{62} \\
-k_{1} N_{1}-k_{61} N_{61}-k_{62} N_{62}-k_{2} N_{2}
\end{array}\right\}+[T]\left\{\begin{array}{l}
p_{1} \\
p_{2} \\
p_{3}
\end{array}\right\}=[T]\left\{\begin{array}{l}
m \ddot{u} \\
m \ddot{v} \\
m \ddot{w}
\end{array}\right\} .
$$


These are the only three governing equations, and the boundary conditions in Eq. (80) reduce to:

Along $x=0, X$ :

$$
\begin{array}{llll}
\delta u=0 & \text { or } & N_{1} T_{11}+N_{61} T_{21} \\
\delta v=0 & \text { or } & N_{1} T_{12}+N_{61} T_{22} \\
\delta w=0 & \text { or } & N_{1} T_{13}+N_{61} T_{23}
\end{array}
$$

Along $y=0, Y$ :

$$
\begin{array}{llll}
\delta u=0 & \text { or } & N_{62} T_{11}+N_{2} T_{21} \\
\delta v=0 & \text { or } & N_{62} T_{12}+N_{2} T_{22}, \\
\delta w=0 & \text { or } & N_{62} T_{13}+N_{2} T_{23}
\end{array}
$$

We note that, if $x$ and/or $y$ are curvilinear, the magnitude of the differential area $d x d y$ may change with $x$ and/or $y$. This area effect is not accounted for in using integration by parts in Eqs. (73) and (76) because it can be done only if the explicit expressions of $x$ and $y$ are known. Next we show how to account for this area effect in using Eqs. (85a) and (86) to obtain the fully nonlinear theory of thin circular plates.

\subsubsection{Circular plate theory}

Figure 2 shows a general axisymmetric membrane described by an orthogonal curvilinear coordinate system $x y z$, where $x$ denotes the meridian (also called the

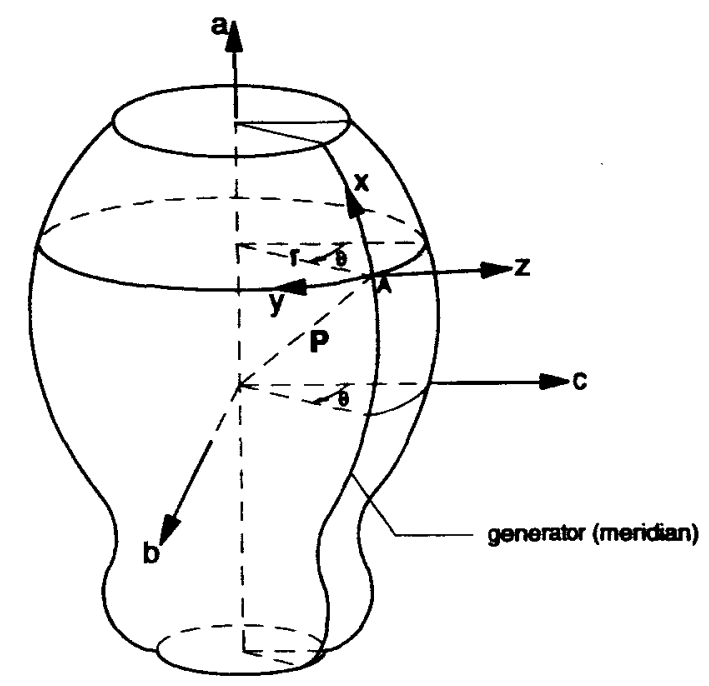

Fig. 2. The two coordinate systems used in describing the undeformed geometry of an axisymmetric membrane. 
generator) and $y$ denotes the circumference. For a circular plate, if the $x$ is chosen to coincide with $r$, we have

$$
d x=d r, \quad d y=r d \theta .
$$

Then, one can follow Eqs. (1)-(4) to show that all initial curvatures are zero except that

$$
k_{4}^{0}=\frac{1}{r} .
$$

It is clear from Eq. (91a) that, if $d \theta$ and $d r$ are fixed, the differential area $d x d y$ changes with $r$. To account for this area effect caused by curvilinear coordinates, one just need to do the following changes for the spatial derivatives of a stress resultant:

$$
\begin{aligned}
& N_{1 x} \rightarrow \frac{d N_{1} r d \theta d r}{d r} \frac{1}{r d \theta d r}=N_{1 r}+N_{1} k_{4}^{0}, \\
& N_{1 y} \rightarrow \frac{d N_{1} r d \theta d r}{r d \theta} \frac{1}{r d \theta d r}=N_{1 \theta} k_{4}^{0} .
\end{aligned}
$$

Hence, the fully nonlinear circular plate theory can be obtained from Eqs. (86), (85a), and (92) as:

$$
\begin{aligned}
& \left\{\begin{array}{c}
N_{1 r}+N_{1} k_{4}^{0}+N_{62 \theta} k_{4}^{0} \\
N_{61 r}+N_{61} k_{4}^{0}+N_{2 \theta} k_{4}^{0} \\
Q_{1 r}+Q_{1} k_{4}^{0}+Q_{2 \theta} k_{4}^{0}
\end{array}\right\}+\left\{\begin{array}{c}
k_{1} Q_{1}-k_{5} N_{61}+k_{62} Q_{2}-k_{4} N_{2} \\
k_{61} Q_{1}+k_{5} N_{1}+k_{2} Q_{2}+k_{4} N_{62} \\
-k_{1} N_{1}-k_{61} N_{61}-k_{62} N_{62}-k_{2} N_{2}
\end{array}\right\}+[T]\left\{\begin{array}{l}
p_{1} \\
p_{2} \\
p_{3}
\end{array}\right\} \\
& \quad=[T]\left\{\begin{array}{l}
m \ddot{u} \\
m \ddot{v} \\
m \ddot{w}
\end{array}\right\},
\end{aligned}
$$

where

$$
\begin{aligned}
Q_{1} & =\frac{1}{1+e_{1}}\left(M_{1 r}+M_{1} k_{4}^{0}+M_{6 \theta} k_{4}^{0}-M_{2} k_{4}-M_{6} k_{5}\right), \\
Q_{2} & =\frac{1}{1+e_{2}}\left(M_{2 \theta} k_{4}^{0}+M_{6 r}+M_{6} k_{4}^{0}+M_{6} k_{4}+M_{1} k_{5}\right) .
\end{aligned}
$$

The boundary conditions are the same as those in Eq. (80) except that $d x$ and $d y$ need to be replaced with $d r$ and $r d \theta$, respectively. Similarly, $d x$ and $d y$ need to be replaced with $d r$ and $r d \theta$ in the nonlinear expressions of $e_{1}, e_{2}, \gamma_{61}, \gamma_{62}, k_{1}, k_{2}$, and $k_{6}\left(=k_{61}+k_{62}\right)$ shown in Eqs. (6), (7), (11a), (11b), and (18).

\subsubsection{Accounting for $\gamma_{6}$}

We point out here that the governing equations (83) and (84) are valid and can be derived using the vector approach only if $\gamma_{6}$ is negligibly small. If $\gamma_{6}$ is not small, substituting Eqs. (43) and (44) into Eq. (72) yields

$$
\delta \Pi=\int_{A}\left(\hat{N}_{1} \delta e_{1}+\hat{N}_{2} \delta e_{2}+\hat{N}_{61} \delta \gamma_{61}+\hat{N}_{62} \delta \gamma_{62}+M_{1} \delta k_{1}+M_{2} \delta k_{2}+M_{6} \delta k_{6}\right) d x d y
$$


where

$$
\begin{aligned}
& \hat{N}_{1} \equiv N_{1} \cos \gamma_{61}+N_{6} \sin \gamma_{61}, \\
& \hat{N}_{2} \equiv N_{2} \cos \gamma_{62}+N_{6} \sin \gamma_{62}, \\
& \hat{N}_{61} \equiv\left(1+e_{1}\right)\left(N_{6} \cos \gamma_{61}-N_{1} \sin \gamma_{61}\right), \\
& \hat{N}_{62} \equiv\left(1+e_{2}\right)\left(N_{6} \cos \gamma_{62}-N_{2} \sin \gamma_{62}\right) .
\end{aligned}
$$

Then, one can follow the same procedures to derive the governing equations. However, Eqs. (19), (20), (24a), and (24b) need to be used for $\delta e_{1}, \delta e_{2}, \delta \gamma_{61}$, and $\delta \gamma_{62}$. The derivation is straightforward, but the governing equations obtained are complicated by $\gamma_{6}$. However, if finite elements are used to discretize the system, $\gamma_{6}$ does not complicate the formulation too much. ${ }^{16}$

Large scientific inflatable membranes are usually constructed from speciallyshaped flat gores using a doubler material at the seams or other methods, which leads to structural models that defy closed-form solutions. Moreover, as materials for scientific membrane structures may exhibit nonlinear viscoelastic response, ${ }^{17}$ the $[\tilde{Q}]$ in Eq. (56) may become functions of strains and strain rates. Hence, the finite element method is more appropriate for analyzing real membrane structures. Furthermore, nonlinear finite element equations can be easily solved using an arc-length iteration method, and they can be used to determine bifurcation points when wrinkling starts and to study bifurcated equilibrium paths.

\section{Axisymmetric Membranes Under Axisymmetric Loading}

Rotationally symmetric membranes are popular in scientific applications because they are easy to manufacture and it is easy to predict and control their inflated shapes. Next we derive the fully nonlinear governing equations of axisymmetric membranes subjected to axisymmetric loading.

From the undeformed geometry of the axisymmetric membrane shown in Fig. 2 and the coordinate systems and unit vectors shown in Fig. 1 we obtain that

$$
\begin{aligned}
\mathbf{P} & =a \mathbf{i}_{a}+r \sin \theta \mathbf{i}_{b}+r \cos \theta \mathbf{i}_{c}, \\
d x & =\sqrt{d a^{2}+d r^{2}}= \pm d a \sqrt{1+r_{a}^{2}}, \quad d y=r d \theta, \\
\mathbf{j}_{1} & =\frac{\partial \mathbf{P}}{\partial x}=\frac{ \pm 1}{\sqrt{1+r_{a}^{2}}}\left(\mathbf{i}_{a}+r_{a} \sin \theta \mathbf{i}_{b}+r_{a} \cos \theta \mathbf{i}_{c}\right), \\
\mathbf{j}_{2} & =\frac{\partial \mathbf{P}}{\partial y}=\cos \theta \mathbf{i}_{b}-\sin \theta \mathbf{i}_{c}, \\
\mathbf{j}_{3} & =\mathbf{j}_{1} \times \mathbf{j}_{2}=\frac{ \pm 1}{\sqrt{1+r_{a}^{2}}}\left(-r_{a} \mathbf{i}_{a}+\sin \theta \mathbf{i}_{b}+\cos \theta \mathbf{i}_{c}\right),
\end{aligned}
$$

where $\mathbf{P}$ is the position vector of point $\mathbf{A}, r$ is a function of $a, r_{a} \equiv \partial r / \partial a$, and the \pm is determined by the sign of $d a / d x$. Substituting Eq. (95) into Eq. (3) we 
obtain

$$
k_{1}^{0}=-\frac{ \pm r_{a a}}{\left(1+r_{a}^{2}\right)^{3 / 2}}, \quad k_{2}^{0}=\frac{ \pm 1}{r \sqrt{1+r_{a}^{2}}}, \quad k_{4}^{0}=\frac{ \pm r_{a}}{r \sqrt{1+r_{a}^{2}}}, \quad k_{61}^{0}=k_{62}^{0}=k_{5}^{0}=0
$$

Since $x$ and $\theta$ are two curvilinear Lagrangian coordinates to be used, one needs to account for the area effect by making the following replacements:

$$
\begin{aligned}
& N_{1 x} \rightarrow \frac{d N_{1} r d \theta d x}{d x} \frac{1}{r d \theta d x}=N_{1 x}+N_{1} k_{4}^{0}, \\
& N_{1 y} \rightarrow \frac{d N_{1} r d \theta d x}{r d \theta} \frac{1}{r d \theta d x}=\frac{1}{r} N_{1 \theta} .
\end{aligned}
$$

The same replacements need to be done for all other spatial derivatives of stress resultants in the governing equations.

For a specific membrane geometry, one can use Eq. (96a) to derive initial curvatures. For example, if it is a parabolic membrane with the apex at the origin of $a b c$ and the focus at $a=\bar{a}$, then

$$
\begin{gathered}
r^{2}=4 a \bar{a}, \quad r_{a}=\frac{2 \bar{a}}{r}, \quad r_{a a}=\frac{-4 \bar{a}^{2}}{r^{3}}, \\
k_{1}^{0}=\frac{4 \bar{a}^{2}}{\left(r^{2}+4 \bar{a}^{2}\right)^{3 / 2}}, \quad k_{2}^{0}=\frac{1}{\sqrt{r^{2}+4 \bar{a}^{2}}}, \quad k_{4}^{0}=\frac{2 \bar{a}}{r \sqrt{r^{2}+4 \bar{a}^{2}}} .
\end{gathered}
$$

If it is a spherical membrane with the center at the origin of $a b c$ and the radius is $R$, then

$$
\begin{gathered}
R^{2}=a^{2}+r^{2}, \quad r_{a}=\frac{-a}{r}, \quad r_{a a}=-\frac{R^{2}}{r^{3}}, \\
k_{1}^{0}=k_{2}^{0}=\frac{1}{R}, \quad k_{4}^{0}=\frac{-\sqrt{R^{2}-r^{2}}}{r R} .
\end{gathered}
$$

Under axisymmetric loading, displacements only happen on the $x z$ plane, and the convected axes $\hat{\xi}$ and $\hat{\eta}$ are always perpendicular to each other. Hence, we have

$$
\begin{aligned}
v & =0, \quad \frac{\partial()}{\partial y}=\frac{\partial()}{r \partial \theta}=0, \\
k_{61} & =k_{62}=k_{5}=0, \\
\mathbf{i}_{\hat{2}} & =\mathbf{i}_{2}=T_{21} \mathbf{j}_{1}+T_{22} \mathbf{j}_{2}+T_{23} \mathbf{j}_{3}=\mathbf{j}_{2}, \\
\mathbf{i}_{\hat{1}} & =\mathbf{i}_{1}=T_{11} \mathbf{j}_{1}+T_{12} \mathbf{j}_{2}+T_{13} \mathbf{j}_{3}=T_{11} \mathbf{j}_{1}+T_{13} \mathbf{j}_{3}, \\
\mathbf{i}_{3} & =\mathbf{i}_{1} \times \mathbf{i}_{2}=-T_{13} \mathbf{j}_{1}+T_{11} \mathbf{j}_{3} .
\end{aligned}
$$

Moreover, because $\mathbf{i}_{\hat{1}}=\mathbf{i}_{1}$ and $\mathbf{i}_{\hat{2}}=\mathbf{i}_{2}$,

$$
\begin{gathered}
\gamma_{61}=\gamma_{62}=\gamma_{6}=B_{12}=0 \\
B_{11}=e_{1}+z\left(k_{1}-k_{1}^{0}\right), \quad B_{22}=e_{2}+z\left(k_{2}-k_{2}^{0}\right) .
\end{gathered}
$$


Equation (98b) indicates that there is no in-plane shear straining in axisymmetric membranes undergoing axisymmetric deformations, and hence $x$ and $y$ are the principle strain axes. Moreover, because of Eq. (98b), one can see from Eq. (73) that $N_{6}$ should disappear from the governing equations. Substituting Eqs. (96a), (98a), and (98b) into Eqs. (14a), (10), (16), (17), and (70) yields

$$
\begin{gathered}
{[T]=\left[\begin{array}{ccc}
T_{11} & 0 & T_{13} \\
0 & 1 & 0 \\
-T_{13} & 0 & T_{11}
\end{array}\right], \quad T_{11}=\frac{1+u_{x}+w k_{1}^{0}}{1+e_{1}}, \quad T_{13}=\frac{w_{x}-u k_{1}^{0}}{1+e_{1}}} \\
{\left[K_{1}\right]=\left[\begin{array}{ccc}
0 & 0 & -k_{1} \\
0 & 0 & 0 \\
k_{1} & 0 & 0
\end{array}\right], \quad\left[K_{2}\right]=\left[\begin{array}{ccc}
0 & k_{4} & 0 \\
-k_{4} & 0 & -k_{2} \\
0 & k_{2} & 0
\end{array}\right]} \\
p_{1}=-p\left(1+e_{1}\right)\left(1+e_{2}\right) T_{13}, \quad p_{2}=0, \quad p_{3}=p\left(1+e_{1}\right)\left(1+e_{2}\right) T_{11}
\end{gathered}
$$

\subsection{Forward analysis without bending stiffness}

Under static axisymmetric loading and without bending stiffness, because of Eqs. (96a), (98a), (98b), and (99a)-(99c), the second equation of (89) becomes a null statcment of $0=0$ and the other two equations become

$$
\begin{gathered}
N_{1 x}-k_{4} N_{2}=m \ddot{u} T_{11}+m \ddot{w} T_{13}, \\
-k_{1} N_{1}-k_{2} N_{2}+p\left(1+e_{1}\right)\left(1+e_{2}\right)=-m \ddot{u} T_{13}+m \ddot{w} T_{11} .
\end{gathered}
$$

Accounting for the area effect by using Eq. (96b) in Eq. (100a) yields

$$
\frac{d N_{1}}{d x}=N_{2} k_{4}-N_{1} k_{4}^{0}+m \ddot{u} T_{11}+m \ddot{w} T_{13} \text {. }
$$

Post-multiplying Eq. (16) by $[T]$ and using Eqs. (99a) and (99b), we obtain that

$$
\begin{aligned}
& \frac{d T_{11}}{d x}=T_{13}\left(k_{1}-k_{1}^{0}\right), \\
& \frac{d T_{13}}{d x}=T_{11}\left(k_{1}^{0}-k_{1}\right) .
\end{aligned}
$$

Moreover, it follows from $\mathrm{Eq} .(99 \mathrm{a})$ that

$$
\begin{gathered}
\frac{d u}{d x}=\left(1+e_{1}\right) T_{11}-w k_{1}^{0}-1, \\
\frac{d w}{d x}=\left(1+e_{1}\right) T_{13}+u k_{1}^{0} .
\end{gathered}
$$

Substituting Eqs. (96a), (98a), and (99a) into Eqs. (7) and (18) we obtain that

$$
e_{2}=u k_{4}^{0}+w k_{2}^{0}, \quad k_{2}=T_{11} k_{2}^{0}-T_{13} k_{4}^{0}, \quad k_{4}=T_{13} k_{2}^{0}+T_{11} k_{4}^{0} .
$$


Because $\gamma_{6}=0$ and the bending stiffnesses are neglected, it follows from Eq. (74b) that

$$
e_{1}=\frac{N_{1}-\tilde{Q}_{12} e_{2}}{\tilde{Q}_{11}}, \quad N_{2}=\tilde{Q}_{21} e_{1}+\tilde{Q}_{22} e_{2} .
$$

Furthermore, we obtain from Eq. (100b) that

$$
k_{1}=\frac{p\left(1+e_{1}\right)\left(1+e_{2}\right)-k_{2} N_{2}+m \ddot{u} T_{13}-m \ddot{w} T_{11}}{N_{1}} .
$$

Substituting Eqs. (102a)-(102c) into Eqs. (101a)-(101e) yields five equations in terms of $N_{1}, T_{11}, T_{13}, u$, and $w$. In other words, Eqs. (101a)-(101e) are the five governing differential equations. However, it is a fourth-order system because it follows from Eq. (90) that the boundary conditions are to specify:

$$
\begin{array}{lll}
u & \text { or } & N_{1} T_{11} \\
w & \text { or } & N_{1} T_{13}
\end{array}
$$

at $x=0$ and $x=L$, where $L$ is the arc length of the meridian. Because $\mathbf{i}_{1}$ is a unit vector, the constraint equation needed is:

$$
T_{11}^{2}+T_{13}^{2}=1
$$

Equations (101a)-(101e) (or Eqs. (101a), (101c)-(101e) with $T_{11}= \pm \sqrt{1-T_{13}^{2}}$ ) will be used to obtain numerically exact solutions by using a multiple shooting method. ${ }^{18}$

One can see from Eq. (96a) that, at $r=0, k_{2}^{0}, k_{4}^{0}, k_{2}$, and $k_{4}$ may become singular and it is impossible to compute $e_{1}, N_{2}$, and $k_{1}$ using Eqs. (102b) and (102c) because $e_{2}, k_{2}$, and $k_{4}$ cannot be computed using Eq. (102a). However, because of the axisymmetric geometry, we have

$$
\begin{aligned}
& T_{11}=1, \quad T_{13}=0, \quad k_{1}^{0}=k_{2}^{0}, \quad k_{2}=k_{1}, \\
& k_{4}=k_{4}^{0}, \quad e_{1}=e_{2}, \quad N_{1}=N_{2}, \quad N_{1 x}=0 .
\end{aligned}
$$

at $r=0$, where $k_{4}=k_{4}^{0}$ is obtained from Eq. (102a) by using $T_{11}=1$ and $T_{13}=0$. Note that $k_{2}$ and $k_{4}$ do not represent actual curvatures and they are functions used for exact description of $\xi$ and $\eta$, as explained right after Eq. (18). Substituting Eq. (105a) into Eqs. (102b) and (102c) yields

$$
e_{1}=\frac{N_{1}}{\tilde{Q}_{11}+\tilde{Q}_{12}}, \quad N_{2}=\left(\tilde{Q}_{21}+\tilde{Q}_{22}\right) e_{1}, \quad k_{1}=\frac{p\left(1+e_{1}\right)^{2}-m \ddot{w}}{2 N_{1}} .
$$

We note that $N_{1}=N_{2}$ in Eqs. $(105 \mathrm{a})$ and $(105 \mathrm{~b})$ results in $\tilde{Q}_{11}=\tilde{Q}_{22}$. In other words, if an initially axisymmetric membrane is not manufactured to have $\tilde{Q}_{11}=\tilde{Q}_{22}$, the membrane will not have an axisymmetric shape under axisymmetric loading.

If a spherical membrane undergoes uniform expansion with respect to its center, we have

$$
u=T_{13}=0, \quad T_{11}=1 .
$$


Because of symmetry, we have

$$
N_{1}=N_{2}, \quad k_{1}=k_{2}, \quad k_{1}^{0}=k_{2}^{0}, \quad w_{x}=N_{1 x}=0 .
$$

Moreover, substituting Eq. (106a) into Eqs. (99a) and (102a) yields

$$
\begin{gathered}
e_{1}=w k_{1}^{0}=e_{2}=w k_{2}^{0}=\frac{w}{R}, \\
k_{2}=k_{2}^{0}, \quad k_{4}=k_{4}^{0} .
\end{gathered}
$$

Equation (106d) is due to the fact that $k_{2}$ and $k_{4}$ are defined with respect to the undeformed length $d x$ and are not the actual deformed curvatures, as explained right after Eq. (18). Equations (106a)-(106d) satisfy exactly the governing equations (101a)-(101e) and make them to be $0=0$. Substituting Eqs. (106b)-(106d) into Eqs. (102b) and (102c) yields the nonlinear function of $p$ in terms of $w$ as:

$$
p=\frac{2\left(\tilde{Q}_{11}+\tilde{Q}_{12}\right) w+R^{2} m \ddot{w}}{(R+w)^{2}}
$$

If $w / R$ is small and the pressure is static, one can replace $R+w$ with $R$ and then $p$ is proportional to $w$.

\subsection{Forward analysis with bending stiffness}

If bending stiffnesses are to be included in the analysis of axisymmetric membranes under axisymmetric loading, the strains shown in Eq. (63) need to be used, instead of Eq. (87). However, the surface analysis shown in Eqs. (95)-(99c) are still valid. Although Eq. (74b) show that $N_{6}$ and $M_{6}$ may be nonzero due to elastic couplings, Eq. (73) shows that $N_{6}$ and $M_{6}$ should not appear in the governing equations because $k_{6}=\gamma_{61}=\gamma_{62}=0$ (see Eqs. (98a) and (98b)). However, $M_{1}$ and $M_{2}$ should be included. Moreover, substituting Eq. (98a) into Eq. (85a) yields $Q_{2}=0$ and

$$
\frac{d M_{1}}{d x}=\left(1+e_{1}\right) Q_{1}+M_{2} k_{4}-M_{1} k_{4}^{0},
$$

where $M_{1 x}$ is replaced with $M_{1 x}+M_{1} k_{4}^{0}$ to account for the area effect, as shown in Eq. (96b). The first and third equations of (86) become

$$
\begin{gathered}
\frac{d N_{1}}{d x}=k_{4} N_{2}-k_{1} Q_{1}-k_{4}^{0} N_{1}+m \ddot{u} T_{11}+m \ddot{w} T_{13}, \\
\frac{d Q_{1}}{d x}=k_{1} N_{1}+k_{2} N_{2}-p\left(1+e_{1}\right)\left(1+e_{2}\right)-k_{4}^{0} Q_{1}-m \ddot{u} T_{13}+m \ddot{w} T_{11},
\end{gathered}
$$

where $N_{1 x}$ and $Q_{1 x}$ are replaced with $N_{1 x}+N_{1} k_{4}^{0}$ and $Q_{1 x}+Q_{1} k_{4}^{0}$, respectively, to account for the area effect. Moreover, Eqs. (101b)-(101e) are still valid and are repeated here as:

$$
\frac{d T_{11}}{d x}=T_{13}\left(k_{1}-k_{1}^{0}\right)
$$




$$
\begin{gathered}
\frac{d T_{13}}{d x}=T_{11}\left(k_{1}^{0}-k_{1}\right), \\
\frac{d u}{d x}=\left(1+e_{1}\right) T_{11}-w k_{1}^{0}-1, \\
\frac{d w}{d x}=\left(1+e_{1}\right) T_{13}+u k_{1}^{0} .
\end{gathered}
$$

Moreover, Eq. (102a) is also valid and is repeated here as:

$$
e_{2}=u k_{4}^{0}+w k_{2}^{0}, \quad k_{2}=T_{11} k_{2}^{0}-T_{13} k_{4}^{0}, \quad k_{4}=T_{13} k_{2}^{0}+T_{11} k_{4}^{0} .
$$

Because $\gamma_{6}=k_{6}=k_{6}^{0}=0$, it follows from Eq. (74b) that

$$
\begin{aligned}
& \tilde{Q}_{11} e_{1}+\tilde{Q}_{14}\left(k_{1}-k_{1}^{0}\right)=N_{1}-\tilde{Q}_{12} e_{2}-\tilde{Q}_{15}\left(k_{2}-k_{2}^{0}\right), \\
& \tilde{Q}_{41} e_{1}+\tilde{Q}_{44}\left(k_{1}-k_{1}^{0}\right)=M_{1}-\tilde{Q}_{42} e_{2}-\tilde{Q}_{45}\left(k_{2}-k_{2}^{0}\right) .
\end{aligned}
$$

Using Cramer's rule we obtain that

$$
\begin{aligned}
& k_{1}=k_{1}^{0}+\frac{\left[M_{1}-\tilde{Q}_{42} e_{2}-\tilde{Q}_{45}\left(k_{2}-k_{2}^{0}\right)\right] \tilde{Q}_{11}-\left[N_{1}-\tilde{Q}_{12} e_{2}-\tilde{Q}_{15}\left(k_{2}-k_{2}^{0}\right)\right] \tilde{Q}_{41}}{\tilde{Q}_{11} \tilde{Q}_{44}-\tilde{Q}_{14}^{2}} \\
& e_{1}=\frac{\left[N_{1}-\tilde{Q}_{12} e_{2}-\tilde{Q}_{15}\left(k_{2}-k_{2}^{0}\right)\right] \tilde{Q}_{44}-\left[M_{1}-\tilde{Q}_{42} e_{2}-\tilde{Q}_{45}\left(k_{2}-k_{2}^{0}\right)\right] \tilde{Q}_{14}}{\tilde{Q}_{11} \tilde{Q}_{44}-\tilde{Q}_{14}^{2}}
\end{aligned}
$$

Moreover, we obtain from Eq. (74b) that

$$
\begin{aligned}
& N_{2}=\tilde{Q}_{21} e_{1}+\tilde{Q}_{22} e_{2}+\tilde{Q}_{24}\left(k_{1}-k_{1}^{0}\right)+\tilde{Q}_{25}\left(k_{2}-k_{2}^{0}\right), \\
& M_{2}=\tilde{Q}_{51} e_{1}+\tilde{Q}_{52} e_{2}+\tilde{Q}_{54}\left(k_{1}-k_{1}^{0}\right)+\tilde{Q}_{55}\left(k_{2}-k_{2}^{0}\right) .
\end{aligned}
$$

Substituting Eqs. (109a)-(109c) into Eqs. (108a)-(108g) yields seven equations in terms of $M_{1}, N_{1}, Q_{1}, T_{11}, T_{13}, u$, and $w$. In other words, Eqs. (108a)-(108g) (or Eqs. (108a)-(108c), (108e)-(108g) with $\left.T_{11}= \pm \sqrt{1-T_{13}^{2}}\right)$ are the governing differential equations. We need the constraint equation (104) because the system is of sixth-order, as shown by the following boundary conditions: to specify

$$
\begin{array}{rll}
u & \text { or } & N_{1} T_{11}-Q_{1} T_{13} \\
w & \text { or } & N_{1} T_{13}+Q_{1} T_{11} \\
T_{11}\left(\text { i.e., } \theta_{2}\right) & \text { or } & M_{1}
\end{array}
$$

at $x=0$ and $x=L$.

Because of the axisymmetric geometry, the singularities at $r=0$ can be dissolved because, at $r=0$,

$$
\begin{gathered}
T_{11}=1, \quad T_{13}=0, \quad k_{1}^{0}=k_{2}^{0}, \quad k_{1}=k_{2}, \quad k_{4}=k_{4}^{0}, \quad e_{1}=e_{2}, \\
N_{1}=N_{2}, \quad M_{1}=M_{2}, \quad M_{1 x}=N_{1 x}=Q_{1}=0 .
\end{gathered}
$$


Using Eq. (111a) in Eq. (109b) we obtain

$$
\begin{aligned}
& e_{1}=\frac{N_{1}\left(\tilde{Q}_{44}+\tilde{Q}_{45}\right)-M_{1}\left(\tilde{Q}_{14}+\tilde{Q}_{15}\right)}{\left(\tilde{Q}_{11}+\tilde{Q}_{12}\right)\left(\tilde{Q}_{44}+\tilde{Q}_{45}\right)-\left(\tilde{Q}_{14}+\tilde{Q}_{15}\right)\left(\tilde{Q}_{41}+\tilde{Q}_{42}\right)} \\
& k_{1}=k_{1}^{0}+\frac{M_{1}\left(\tilde{Q}_{11}+\tilde{Q}_{12}\right)-N_{1}\left(\tilde{Q}_{41}+\tilde{Q}_{42}\right)}{\left(\tilde{Q}_{11}+\tilde{Q}_{12}\right)\left(\tilde{Q}_{44}+\tilde{Q}_{45}\right)-\left(\tilde{Q}_{14}+\tilde{Q}_{15}\right)\left(\tilde{Q}_{41}+\tilde{Q}_{42}\right)} .
\end{aligned}
$$

Using Eq. (111a) in Eq. (109c) we obtain

$$
\begin{aligned}
& N_{2}=\left(\tilde{Q}_{21}+\tilde{Q}_{22}\right) e_{1}+\left(\tilde{Q}_{24}+\tilde{Q}_{25}\right)\left(k_{1}-k_{1}^{0}\right), \\
& M_{2}=\left(\tilde{Q}_{51}+\tilde{Q}_{52}\right) e_{1}+\left(\tilde{Q}_{54}+\tilde{Q}_{55}\right)\left(k_{1}-k_{1}^{0}\right)
\end{aligned}
$$

If the material is isotropic, it follows from Eq. (98b) that the elastic energy $\Pi$ is given by:

$$
\begin{aligned}
\Pi & =\frac{1}{2} \int_{A} \int_{-h / 2}^{h / 2}\left\{\begin{array}{l}
B_{11} \\
B_{22}
\end{array}\right\}^{T}\left\{\begin{array}{l}
J_{11} \\
J_{22}
\end{array}\right\} d x d y d z \\
& =\frac{1}{2} \int_{A}\left(\left\{\begin{array}{l}
e_{1} \\
e_{2}
\end{array}\right\}^{T}[\bar{Q}]\left\{\begin{array}{l}
e_{1} \\
e_{2}
\end{array}\right\} h+\left\{\begin{array}{l}
k_{1}-k_{1}^{0} \\
k_{2}-k_{2}^{0}
\end{array}\right\}^{T}[\bar{Q}]\left\{\begin{array}{l}
k_{1}-k_{1}^{0} \\
k_{2}-k_{2}^{0}
\end{array}\right\} \frac{h^{3}}{12}\right) 2 \pi r d x \\
{[\bar{Q}] } & \equiv \frac{E}{1-\nu^{2}}\left[\begin{array}{ll}
1 & \nu \\
\nu & 1
\end{array}\right] .
\end{aligned}
$$

Here the first term is due to in-plane stretching and the second term is due to bending.

\subsection{Eigenvalue analysis}

To derive linear natural frequencies and mode shapes of vibration with respect to a deformed static equilibrium configuration we assume

$$
u=\bar{u}+\bar{u}, \quad w=\bar{w}+\tilde{w},
$$

where $\bar{u}$ and $\bar{w}$ denote large static displacements, and $\tilde{u}$ and $\tilde{w}$ denote small dynamic displacements. If the dynamic displacements are assumed to be harmonic at a natural frequency $\omega$, we have

$$
\ddot{u}=-\omega^{2} \tilde{u}, \quad \ddot{w}=-\omega^{2} \tilde{w} .
$$

Substituting Eqs. (113) and (114) into Eqs. (101a)-(101e) and (102a)-(102c) and using Taylor's expansions yields the following first-order expansions:

$$
\begin{gathered}
\frac{d \tilde{N}_{1}}{d x}=\tilde{N}_{2} \bar{k}_{4}+\bar{N}_{2} \tilde{k}_{4}-\tilde{N}_{1} k_{4}^{0}-m \tilde{u} \omega^{2} \bar{T}_{11}-m \tilde{w} \omega^{2} \bar{T}_{13}, \\
\frac{d \tilde{T}_{11}}{d x}=\tilde{T}_{13}\left(\bar{k}_{1}-k_{1}^{0}\right)+\bar{T}_{13} \tilde{k}_{1},
\end{gathered}
$$




$$
\begin{gathered}
\frac{d \tilde{T}_{13}}{d x}=\tilde{T}_{11}\left(k_{1}^{0}-\bar{k}_{1}\right)-\bar{T}_{11} \tilde{k}_{1}, \\
\frac{d \tilde{u}}{d x}=\left(1+\bar{e}_{1}\right) \tilde{T}_{11}+\tilde{e}_{1} \bar{T}_{11}-\tilde{w} k_{1}^{0}, \\
\frac{d \tilde{w}}{d x}=\left(1+\bar{e}_{1}\right) \tilde{T}_{13}+\tilde{e}_{1} \bar{T}_{13}+\tilde{u} k_{1}^{0}, \\
\frac{d \omega}{d x}=0,
\end{gathered}
$$

and

$$
\begin{gathered}
\tilde{e}_{2}=\tilde{u} k_{4}^{0}+\tilde{w} k_{2}^{0}, \quad \tilde{k}_{2}=\tilde{T}_{11} k_{2}^{0}-\tilde{T}_{13} k_{4}^{0}, \quad \tilde{k}_{4}=\tilde{T}_{13} k_{2}^{0}+\tilde{T}_{11} k_{4}^{0}, \\
\tilde{e}_{1}=\frac{\tilde{N}_{1}-\tilde{Q}_{12} \tilde{e}_{2}}{\tilde{Q}_{11}}, \quad \tilde{N}_{2}=\tilde{Q}_{21} \tilde{e}_{1}+\tilde{Q}_{22} \tilde{e}_{2} \\
\tilde{k}_{1}=\frac{p\left(\tilde{e}_{1}+\tilde{e}_{2}+\bar{e}_{1} \tilde{e}_{2}+\tilde{e}_{1} \bar{e}_{2}\right)-\bar{k}_{2} \tilde{N}_{2}-\tilde{k}_{2} \bar{N}_{2}+m \tilde{w} \omega^{2} \bar{T}_{11}-\bar{k}_{1} \tilde{N}_{1}}{\bar{N}_{1}}
\end{gathered}
$$

where Eq. (115f) is based on the fact that the natural frequency $\omega$ is the same for every point on the membrane. At $r=0$, it follows from Eqs. (113), (114), and (105b) that

$$
\begin{aligned}
& \tilde{e}_{1}=\frac{\tilde{N}_{1}}{\tilde{Q}_{11}+\tilde{Q}_{12}}, \quad \tilde{N}_{2}=\tilde{N}_{1}, \\
& \tilde{k}_{1}=\frac{p\left(\tilde{e}_{1}+\bar{e}_{1} \tilde{e}_{1}\right)+\left(m \tilde{w} \omega^{2} / 2\right)-\bar{k}_{1} \tilde{N}_{1}}{\bar{N}_{1}} .
\end{aligned}
$$

Substituting Eqs. (116a)-(116c) (or Eq. (117)) into Eqs. (115a)-(115f) yields six equations in terms of $\tilde{N}_{1}, \tilde{T}_{11}, \tilde{T}_{13}, \tilde{u}, \tilde{w}$, and $\omega$. Because $\left(\bar{T}_{11}+\tilde{T}_{11}\right)^{2}+\left(\bar{T}_{13}+\tilde{T}_{13}\right)^{2}=1$ and $\bar{T}_{11}^{2}+\bar{T}_{13}^{2}=1$, the linear expansion of $\tilde{T}_{11}$ is $\tilde{T}_{11}=-\tilde{T}_{13} \bar{T}_{13} / \bar{T}_{11}$. Hence, only Eqs. $(115 \mathrm{a}),(115 \mathrm{c})-(115 \mathrm{f})$ need to be solved in the multiple shooting process.

\subsection{Inverse analysis}

In the design of regular structures (e.g., building and aircraft structures), the deformed shape is usually not specified but is put as an inequality constraint in the design optimization process. Hence, the final design can be obtained by trying different undeformed shapes and dimensions to satisfy all equality and inequality constraints, which is not really an inverse problem. On the other hand, a precision membrane is usually required to have a precise inflated (or deformed) shape with a 


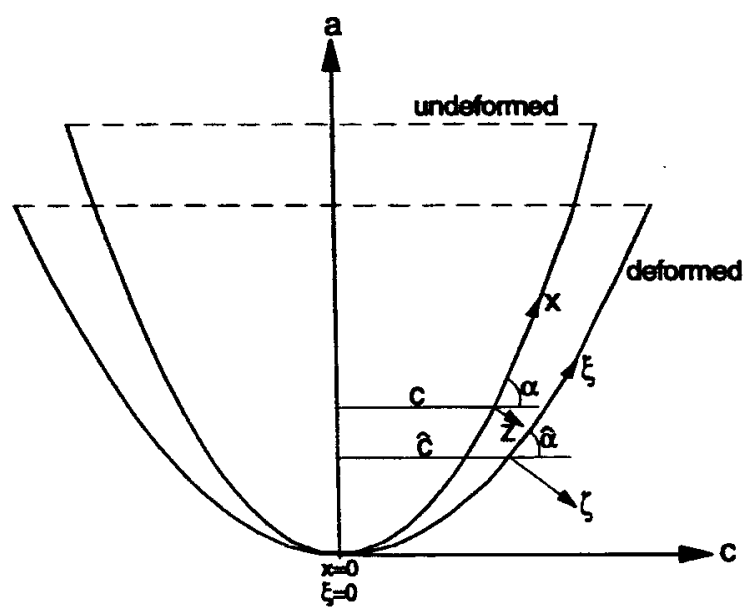

Fig. 3. The generators of the undeformed and deformed shapes of an axisymmetric membrane.

known inflation pressure, but the undeformed shape is unknown. Hence, the design of a precision membrane is a fully inverse problem. Next we show how to solve such a fully inverse problem.

Figure 3 shows how the deformed shape of an axisymmetric membrane is related to its unknown undeformed shape. Because of numerical difficulties in using coordinates $a$ or $c(=r)$ as the independent variable, we will use the curvilinear coordinate $\xi$ as the independent variable. Because

$$
\left(1+e_{1}\right) d x=d \xi, \quad\left(1+e_{2}\right) d y=d \eta,
$$

it follows from Figs. 2 and 3 and the definitions of $k_{1}^{0}, k_{2}^{0}, k_{1}$, and $k_{2}$ shown in Eq. (18) that

$$
\begin{gathered}
k_{1}^{0}=\frac{\partial \alpha}{\partial x}, \quad k_{1}=\frac{\partial \hat{\alpha}}{\partial x}=\left(1+e_{1}\right) \frac{\partial \hat{\alpha}}{\partial \xi}, \\
k_{2}^{0}=\frac{\sin \alpha}{c}, \quad k_{2}=\frac{\sin \hat{\alpha}}{\hat{c}}\left(1+e_{2}\right) .
\end{gathered}
$$

Moreover, because $N_{1}$ represents the tension force per unit of $d y$ and $N_{2}$ represents the tension force per unit of $d x$, we obtain from Eq. (118) that

$$
N_{1}=\hat{N}_{1}\left(1+e_{2}\right), \quad N_{2}=\hat{N}_{2}\left(1+e_{1}\right),
$$

where $\hat{N}_{1}$ represents the tension force per unit of $d \eta$ and $\hat{N}_{2}$ represents the tension force per unit of $d \xi$. From the free-body diagram of the bottom part of the deformed membrane cutting by a plane parallel to the $c$ axis (see Fig. 3), because the internal pressure is balanced by the membrane force $\hat{N}_{1}$, we obtain the following equilibrium equation:

$$
p \hat{c}^{2} \pi=\hat{N}_{1} 2 \pi \hat{c} \sin \hat{\alpha}
$$


On the other hand, Eq. (100b) is an equilibrium equation for a differential membrane element at any location. Substituting Eqs. (119)-(121) into Eq. (100b) and assuming static deformation (i.e., $\ddot{u}=\ddot{w}=0$ ) yields

$$
p=\frac{\partial \hat{\alpha}}{\partial \xi} \hat{N}_{1}+\frac{\sin \hat{\alpha}}{\hat{c}} \hat{N}_{2} .
$$

Because the pressure and the deformed shape are assumed to be known and hence $p, \hat{\alpha}(\xi)$, and $\hat{c}(\xi)$ are known, $\hat{N}_{1}(\xi)$ and $\hat{N}_{2}(\xi)$ can be solved from Eqs. (122) and (123) as:

$$
\begin{aligned}
& \hat{N}_{1}=\frac{p \hat{c}}{2 \sin \hat{\alpha}} \\
& \hat{N}_{2}=\frac{\hat{c}}{\sin \hat{\alpha}}\left(p-\frac{\partial \hat{\alpha}}{\partial \xi} \hat{N}_{1}\right) .
\end{aligned}
$$

It follows from Eqs. (121) and (56) that

$$
\begin{aligned}
& \hat{N}_{1}\left(1+e_{2}\right)=\tilde{Q}_{11} e_{1}+\tilde{Q}_{12} e_{2}, \\
& \hat{N}_{2}\left(1+e_{1}\right)=\tilde{Q}_{12} e_{1}+\tilde{Q}_{22} e_{2} .
\end{aligned}
$$

Hence, $e_{1}(\xi)$ and $e_{2}(\xi)$ can be obtained from Eq. (125) to be

$$
\begin{aligned}
& e_{1}=\frac{\hat{N}_{1} \tilde{Q}_{22}-\hat{N}_{2}\left(\tilde{Q}_{12}-\hat{N}_{1}\right)}{\tilde{Q}_{11} \tilde{Q}_{22}-\left(\tilde{Q}_{12}-\hat{N}_{1}\right)\left(\tilde{Q}_{12}-\hat{N}_{2}\right)}, \\
& e_{2}=\frac{\hat{N}_{2} \tilde{Q}_{11}-\hat{N}_{1}\left(\tilde{Q}_{12}-\hat{N}_{2}\right)}{\tilde{Q}_{11} \tilde{Q}_{22}-\left(\tilde{Q}_{12}-\hat{N}_{1}\right)\left(\tilde{Q}_{12}-\hat{N}_{2}\right)} .
\end{aligned}
$$

Because the circumferential strain $e_{2}$ at $r=\hat{c}$ should be constant due to symmetry, we have

$$
e_{2}=\frac{\hat{c}}{c}-1
$$

Hence, $c(\xi)$ can be obtained to be

$$
c=\frac{\hat{c}}{1+e_{2}} .
$$

Moreover, it follows from Eq. (118) that

$$
e_{1}=\frac{d \xi}{d x}-1
$$

Hence, $x(\xi)$ can be obtained by performing the following integration:

$$
x=\int_{0}^{\xi} \frac{1}{1+e_{1}} d \xi
$$

It follows from Fig. 3 and Eq. (118) that

$$
d a= \pm \sqrt{d x^{2}-d c^{2}}= \pm \sqrt{1-\left(\frac{d c}{d x}\right)^{2}} d x= \pm \sqrt{1-\left(\left(1+e_{1}\right) \frac{d c}{d \xi}\right)^{2}} \frac{d \xi}{1+e_{1}}
$$


where the sign is determined by the sign of $\sin \hat{\alpha}$. Hence, $a(\xi)$ can be obtained by performing the following integration:

$$
a=\int_{0}^{\xi} \pm \sqrt{\frac{1}{\left(1+e_{1}\right)^{2}}-\left(\frac{d c}{d \xi}\right)^{2}} d \xi
$$

where $a(0)=0$ is assumed. After $a(\xi), c(\xi)$, and $x(\xi)$ are obtained from Eqs. (132), (128), and (130), one can easily obtain the undeformed geometry for manufacturing use. We note that it is more convenient to obtain the $d c / d \xi$ in Eq. (132) by numerical differentiation.

\section{Numerical Results}

Large static deformation analysis, linear eigenvalue analysis, and nonlinear inverse analysis of several different membranes are performed and discussed in this section. For all cases we consider axisymmetric membranes made of Kapton film that has

$$
\begin{gathered}
E=5.51581 \mathrm{GPa}(800 \mathrm{kpsi}), \quad \nu=0.3, \quad \rho=1390 \mathrm{~kg} / \mathrm{m}^{3}, \\
h=0.0127 \mathrm{~mm}(0.0005 \mathrm{in}) .
\end{gathered}
$$

\subsection{Forward analysis}

Inclusion of bending stiffness in membrane analysis results in difficulties in solution convergence in the multiple shooting process because $M_{1}$ and $Q_{1}$ have very small values. Figure 4(a) shows the undeformed (broken line) and deformed (solid and dotted lines) shapes of an axisymmetric parabolic membrane (see Eq. (97a)) having $\bar{a} / L=20,0 \leq x \leq L=1.5 \mathrm{~m}$ and $h=15 \mathrm{~mm}$, being subjected to an internal pressure of $500 \mathrm{~Pa}$, and having a fixed rim at $x=L$. The boundary conditions for Eqs. (108a)-(108c), (108e)-(108g) are

$$
Q_{1}=T_{13}=u=0 \text { at } x=0, \quad M_{1}=u=w=0 \text { at } x=L .
$$

Although Fig. 4(a) shows that the bending stiffness does not cause dramatic change of the deformed geometry, but Fig. $4(\mathrm{~b})$ shows that $N_{1}$ and $N_{2}$ are significantly changed by the bending stiffness. Moreover, Figs. 4 (c) and 4(d) show that $M_{1}$, $M_{2}$, and $Q_{1}$ are also significant. However the elastic energy ratio $R_{e}$ (= bending strain energy/total strain energy) is obtained using Eq. (112) to be $10.6 \%$ for this thick thickness case. If $\bar{a} / L=20, p=500 \mathrm{~Pa}$, and $h=15,10,5,3 \mathrm{~mm}, R_{e}$ is computed to be $10.60 \%, 5.08 \%, 1.30 \%, 0.40 \%$, respectively. We note that $R_{e}$ decreases significantly when $h$ decreases, and it becomes more difficult to obtain a convergent solution when $R_{e}<1.0 \%$. If $\bar{a} / L=10, h=10 \mathrm{~mm}$, and $p=500,400,300,200,100$, $10,1 \mathrm{~Pa}, R_{e}$ is computed to be $2.69 \%, 2.79 \%, 2.91 \%, 3.06 \%, 3.25 \%, 3.47 \%, 3.50 \%$, respectively. We note that $R_{e}$ increases slightly when $p$ decreases. If $h=10 \mathrm{~mm}$, $p=200 \mathrm{~Pa}$, and $\bar{a} / L=20,15,10,5,1, R_{e}$ is computed to be $6.03 \%, 4.39 \%, 3.06 \%$, $2.13 \%, 1.41 \%$, respectively. In other words, $R_{e}$ decreases when $\vec{a}$ decreases. Hence 


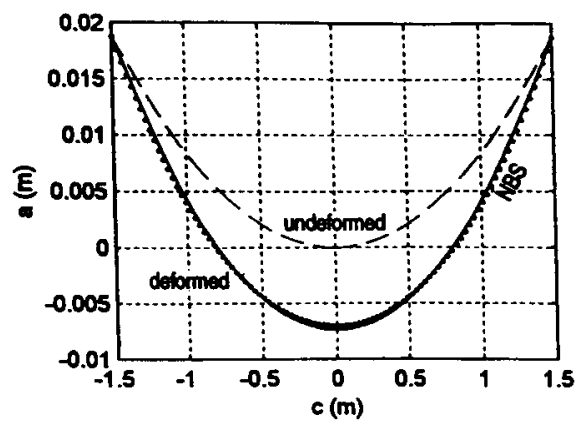

(a)

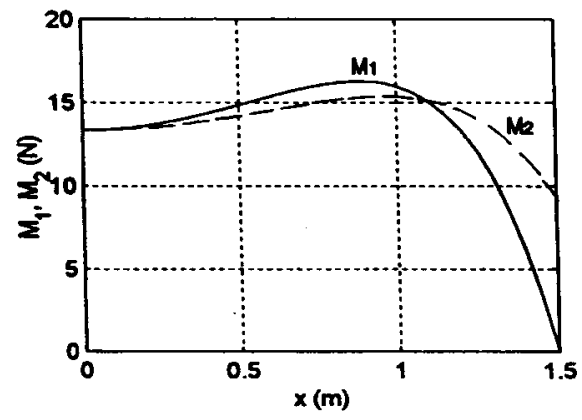

(c)

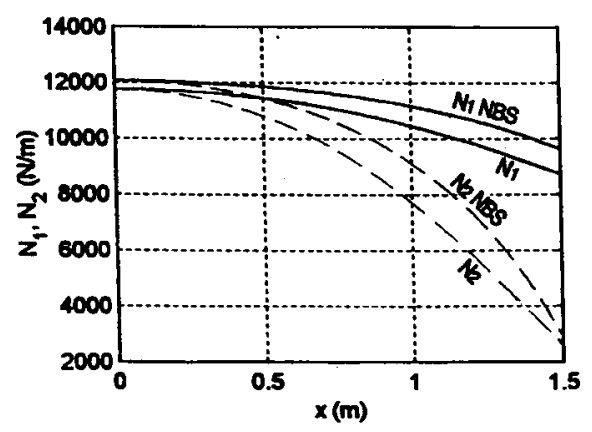

(b)

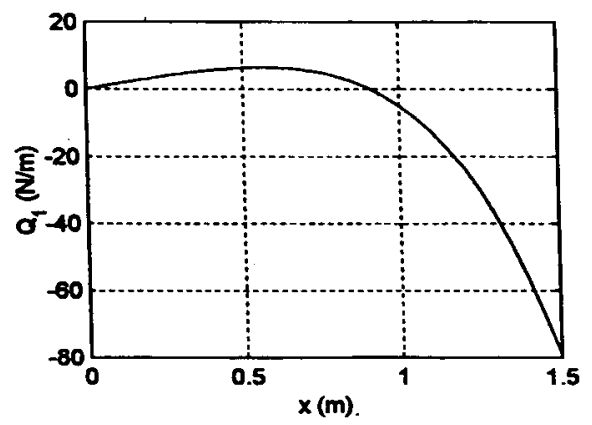

(d)

Fig. 4. The deformation of an axisymmetric parabolic shell with $\bar{a}=20 L, h=15 \mathrm{~mm}$, and $p=$ $500 \mathrm{~Pa}$ : (a) deformed geometries with $(-)$ and without $(\cdots)$ bending stiffness (NBS $=$ no bending stiffness), (b) distributions of $N_{1}$ and $N_{2}$, (c) distributions of $M_{1}$ and $M_{2}$, and (d) distribution of $Q_{1}$.

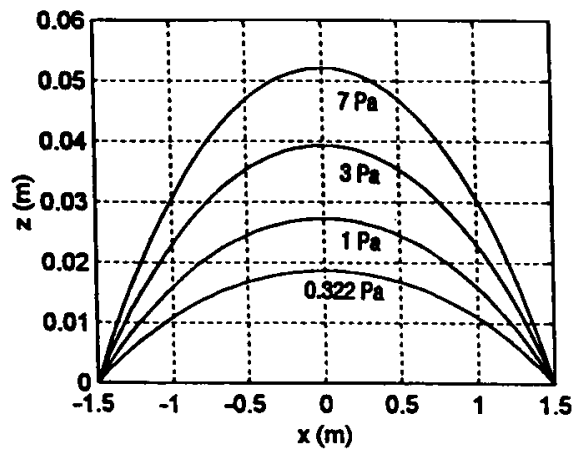

(a)

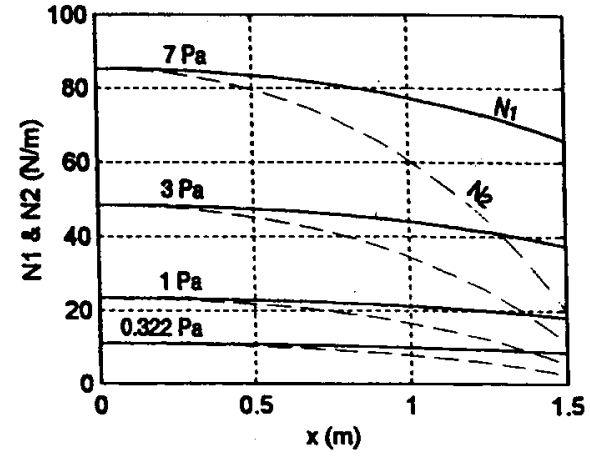

(b)

Fig. 5. The deformation of a circular membrane having $R=1.5 \mathrm{~m}$ and a fixed outer rim and being under different pressures: (a) deformed geometries and (b) distributions of $N_{1}$ and $N_{2}$. 
the bending effect is negligible especially when $h$ and/or $\bar{a}$ are small. Because we consider only membranes made of the thin Kapton film shown in Eq. (133), bending stiffness will be neglected in all following cases and Eqs. (101a), (101c)-(101e) will be used in the forward analysis.

\subsubsection{Circular membrane}

For a circular membrane having a radius $R=1.5 \mathrm{~m}$ and a fixed outer rim, Fig. 5 shows the deformed shapes and the distributions of $N_{1}$ and $N_{2}$ corresponding to different pressures. The corresponding boundary conditions are:

$$
T_{13}=u=0 \text { at } x=0, \quad u=w=0 \text { at } x=R .
$$

The multiple shooting solution is obtained using 21 shooting points. For $p=$ $0.322 \mathrm{~Pa}, w(0)$ is obtained to be $18.6573 \mathrm{~mm}$, which agrees well with the

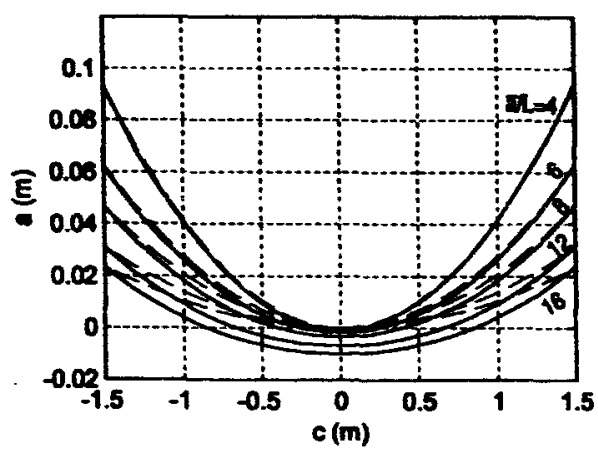

(a)

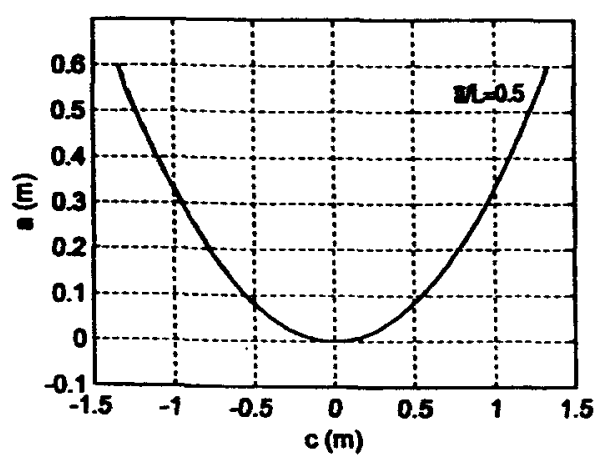

(c)

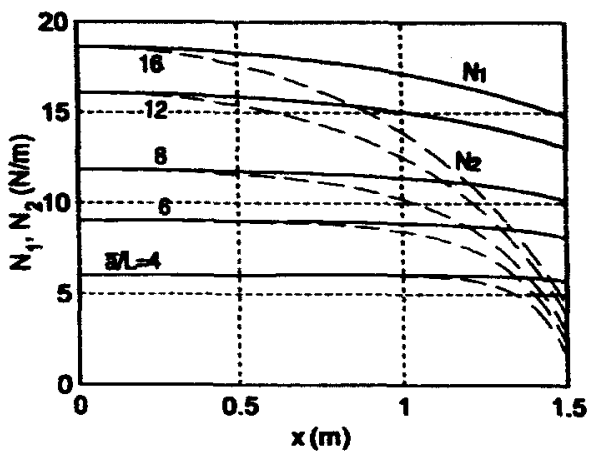

(b)

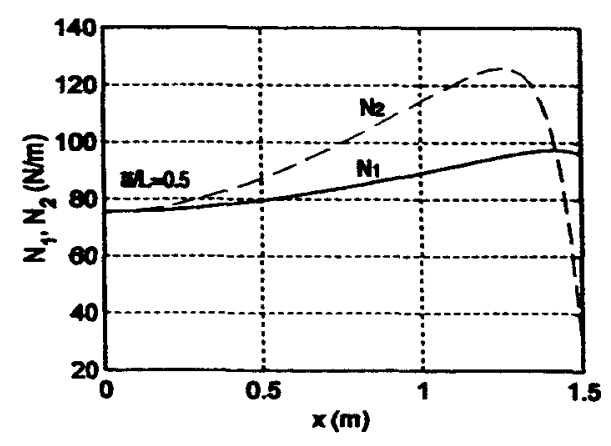

(d)

Fig. 6. The deformation of axisymmetric membranes whose undeformed shapes are parabolic: (a) deformed and undeformed geometries with $p=1 \mathrm{~Pa}$ and different $\bar{a} / L$, (b) distributions of $N_{1}$ and $N_{2}$ with $p=1 \mathrm{~Pa}$ and different $\bar{a} / L,(\mathrm{c})$ deformed and undeformed geometries with $p=100 \mathrm{~Pa}$ and $\bar{a} / L=0.5$, and (d) distributions of $N_{1}$ and $N_{2}$ with $p=100 \mathrm{~Pa}$ and $\bar{a} / L=0.5$. 
MSC/NASTRAN solution, $18.6589 \mathrm{~mm}$, using 50 membrane elements and the solution, $18.6610 \mathrm{~mm}$, using a different formulation and an integration method. ${ }^{9} \mathrm{We}$ note that MSC/NASTRAN uses Green strains and second Piola-Kirchhoff stresses.

\subsubsection{Paraboloidal membrane}

Figure 6(a) shows the undeformed (broken line) and deformed (solid line) shapes of axisymmetric membranes having parabolic undeformed shapes with different focus lengths (i.e., $\bar{a}$ ), $0 \leq x \leq L=1.5 \mathrm{~m}$, and a fixed rim at $x=L$, and being subjected to an internal pressure of $1.0 \mathrm{~Pa}$. The boundary conditions are given by:

$$
T_{13}=u=0 \text { at } x=0, \quad u=w=0 \text { at } x=L .
$$

We note that, under the same pressure, the deformation $w(0)$ increases with $\bar{a}$ and hence the deformed geometry deviates more from a paraboloidal surface. Figure 6(b) shows the distributions of the internal force intensities $N_{1}$ (along $\xi$ ) and $N_{2}$ (along $\eta$ ). We note that $N_{1}>N_{2}, N_{1}=N_{2}$ around the apex, and the area where $N_{1}$ is close to $N_{2}$ increases when $\bar{a}$ decreases. However, Figs. 6(c) and 6(d) show that $N_{2}$ can be larger than $N_{1}$ if $\bar{a}$ is small and/or $p$ is large.

\subsubsection{Toroidal membrane}

We consider a toroidal membrane whose undeformed cross-sectional radius is $R$ $(=3 / \pi \mathrm{m})$ and the center of the cross section is at $c=C_{0}=10 \mathrm{~m}$, as shown in Fig. 7(a). It follows from Fig. 7(a) that

$$
\begin{gathered}
R^{2}=a^{2}+\left(r-C_{0}\right)^{2}, \quad a=R \sin \left(\alpha-\frac{\pi}{2}\right), \quad r=C_{0}+R \cos \left(\alpha-\frac{\pi}{2}\right), \\
\alpha=\frac{x}{R}+\frac{\pi}{2}, \quad 0 \leq x \leq L=3 \mathrm{~m} .
\end{gathered}
$$

We note that the meridian of the toroidal membrane starts from the point $(a, c)=$ $\left(0, C_{0}+R\right)$. It follows from Eqs. (137) and (96a) that

$$
r_{a}=\frac{a}{C_{0}-r}, \quad r_{a a}=\frac{R^{2}}{\left(C_{0}-r\right)^{3}}, \quad k_{1}^{0}=\frac{1}{R}, \quad k_{2}^{0}=\frac{r-C_{0}}{R r}, \quad k_{4}^{0}=\frac{-a}{R r} .
$$

Figures $7(\mathrm{~b})$ and $7(\mathrm{c})$ show the deformed geometry of the right cross section and the distributions of $N_{1}$ and $N_{2}$ when $p=3000 \mathrm{~Pa}$. The RMS of the deviation of the deformed cross section from a fitted circle $0.2 \%$ of $R$, and this ratio increases with $p$. In other words, when $p$ increases, the deformed cross section deviates more from a circle.

\subsection{Eigenvalue analysis}

To understand how pressurization changes the natural frequencies of a membrane structure we consider a parabolic membrane having $L=1.5 \mathrm{~m}$ and $\bar{a}=6 L$ and perform eigenvalue analysis using the method shown in Sec. 3.3. Figure 8(a) shows 


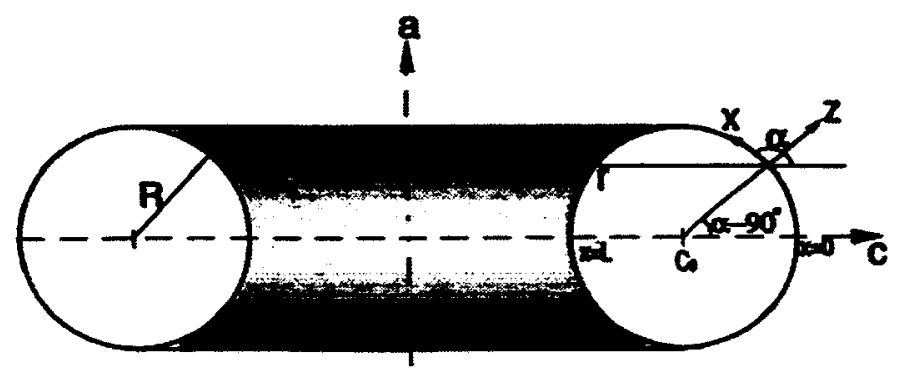

(a)

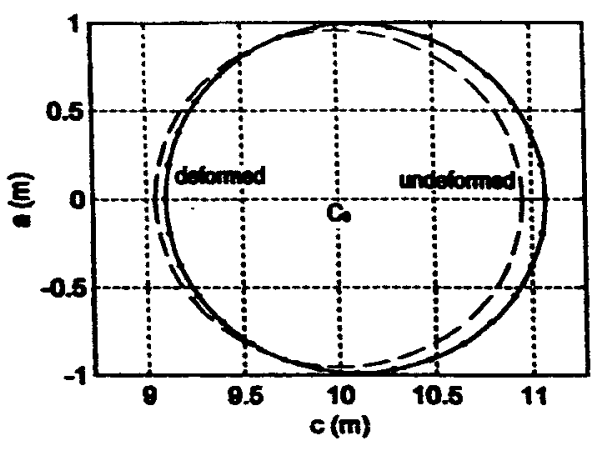

(b)

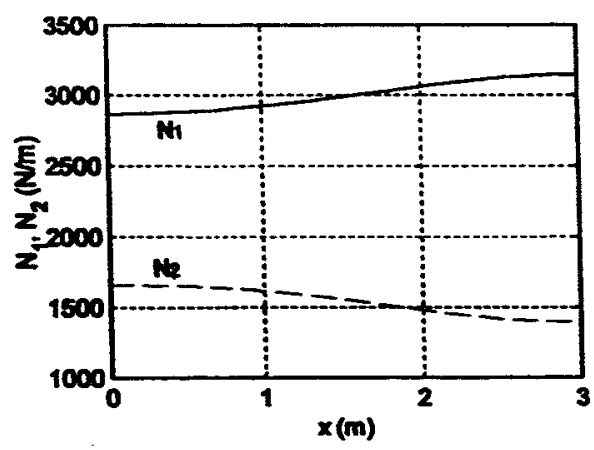

(c)

Fig. 7. The deformation of a toroidal membrane with $C_{0}=10 \mathrm{~m}, L=R \pi=3 \mathrm{~m}$, and $p=$ $3000 \mathrm{~Pa}$ : (a) the undeformed geometry, (b) deformed and undeformed geometries of the cross section, and (c) distributions of $N_{1}$ and $N_{2}$.

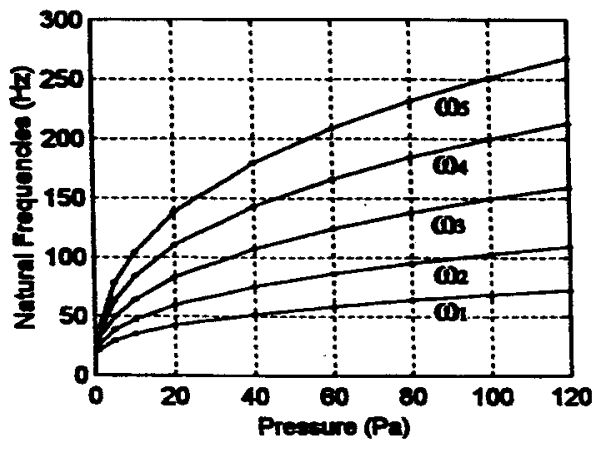

(a)

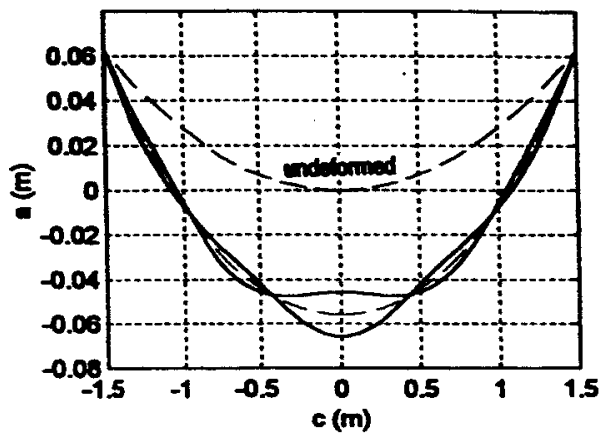

(b)

Fig. 8. Linear natural frequencies and mode shapes of a pressurized parabolic membrane with $L=1.5 \mathrm{~m}$ and $\bar{a}=6 L$ : (a) the first five natural frequencies, and (b) the third mode shape when $p=60 \mathrm{~Pa}$. 
the influence of the internal pressure $p$ on the first five natural frequencies. It is apparent that natural frequencies increase about quadratically with $p$ and the increment rate is high when $p$ is small. Figure $8(\mathrm{~b})$ shows the third linear mode shape when $p=60 \mathrm{~Pa}$. As the derivation is for axisymmetric deformation, only axisymmetric vibration modes can be obtained here. To obtain nonaxisymmetric modes one needs to solve the equations derived in Sec. 2.3 using the finite element method or other methods.

In the formulations shown in Sec. 3.3 the change of $p$ due to the small displacements $\tilde{u}$ and $\tilde{w}$ was neglected and it was assumed that $p=\vec{p}+\tilde{p} \approx \bar{p}$. However, it is necessary to include $\tilde{p}$ in the calculation of low-order natural frequencies (especially the first-mode frequency) of some types of membranes. For example, if a pressurized spherical membrane undergoes the breathing-mode vibration (i.e., the first axisymmetric mode) and the gas inside the membrane is assumed to be an ideal gas, one can use $\bar{p}(R+\bar{w})^{3} 4 \pi / 3=p(R+\bar{w}+\tilde{w})^{3} 4 \pi / 3$ to derive the linear approximation of $p$ as $p=\bar{p}(1-3 \tilde{w} /(R+\bar{w}))$. Hence, one can use Eq. (107) to derive the first axisymmetric-mode frequency $\omega_{1}$ to be

$$
\omega_{1}=\sqrt{\frac{2\left(\tilde{Q}_{11}+\tilde{Q}_{12}\right)+\bar{p}(R+\bar{w})}{m R^{2}}} .
$$

If $\tilde{p}$ is not included, $\omega_{1}=\sqrt{\left(2\left(\tilde{Q}_{11}+\tilde{Q}_{12}\right)-2 \vec{p}(R+\bar{w})\right) /\left(m R^{2}\right)}$, which is erroneous. However, $\tilde{p}$ is negligible for high-frequency modes because of their wavy shapes (e.g., Fig. 8(b)).

\subsection{Inverse analysis}

\subsubsection{Spherical membrane}

To verify the inverse analysis method shown in Sec. 3.4 we consider a deformed spherical membrane whose deformed radius is $\hat{R}(=3 / \pi \mathrm{m})$ and whose material is shown in Eq. (133). It follows from Fig. 3 that

$$
\hat{a}=\hat{R}(1-\cos \hat{\alpha}), \quad \hat{c}=\hat{R} \sin \hat{\alpha}, \quad \hat{\alpha}=\frac{\xi}{\hat{R}}, \quad \frac{\partial \hat{\alpha}}{\partial \xi}=\frac{1}{\hat{R}}, \quad 0 \leq \xi \leq 3 \mathrm{~m} .
$$

If $p=10000 \mathrm{~Pa}$, the undeformed geometry (broken line) obtained from the deformed geometry (solid line) is shown in Fig. $9(\mathbf{a})$. The exact solution of $w(\hat{R} \pi)$ can be obtained from Eq. (107) with $\ddot{w}=0$ to be

$$
w(\hat{R} \pi)=\hat{a}(\hat{R} \pi)-a(\hat{R} \pi)=\frac{p \hat{R}^{2}}{\tilde{Q}_{11}+\tilde{Q}_{12}} .
$$

The inverse solution of $w(\hat{R} \pi)$ is $0.04553136 \mathrm{~m}$, which is less than the exact solution (141) by $0.067 \%$. Figure 9 (a) shows also that the obtained undeformed geometry fits perfectly with a circle (dots). Moreover, Fig. 9 (b) shows that $N_{2}=N_{1}$, as it should be. Furthermore, we use the obtained undeformed geometry and the forward 


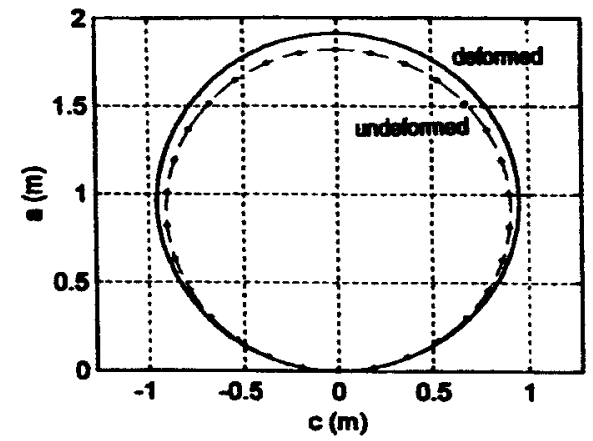

(a)

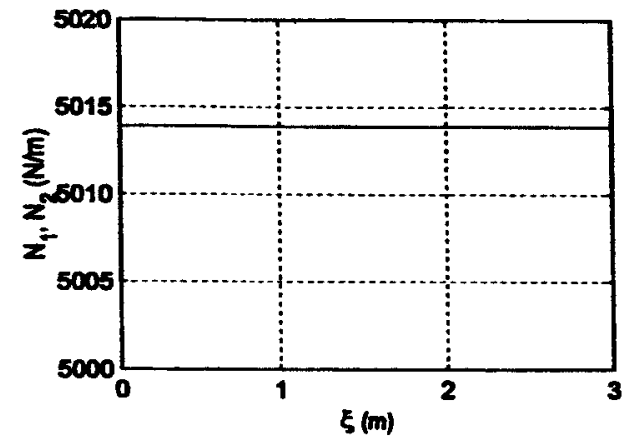

(b)

Fig. 9. The inverse analysis of a known deformed spherical membrane with $L=\hat{R} \pi=3 \mathrm{~m}$ and $p=10000 \mathrm{~Pa}:$ (a) deformed $(-)$, inverse undeformed (---), and circle-fitted undeformed ( $\cdots)$ geometries, and (b) distributions of $N_{1}$ and $N_{2}$.

analysis method to compute the deformed geometry, and the obtained deformed geometry overlaps with the initial deformed geometry. This verifies the proposed inverse analysis method.

\subsubsection{Paraboloidal membrane}

Because of their broad applications in communication, axisymmetric membranes having a deformed paraboloidal surface are important membranes. Next we consider an axisymmetric membrane whose deformed shape is parabolic and is given by:

$$
\hat{c}^{2}=4 \bar{a} \hat{a} \text {. }
$$

From Fig. 3 we obtain

$$
d \xi=\sqrt{d \hat{c}^{2}+d \hat{a}^{2}}=\sqrt{1+\left(\frac{d \hat{a}}{d \hat{c}}\right)^{2}} d \hat{c} .
$$

It follows from Eqs. (142) and (143) and integration that $\xi$ and $\hat{c}$ are related as:

$$
\xi=\frac{\hat{c}}{4} \sqrt{4+\frac{\hat{c}^{2}}{\bar{a}^{2}}}+\bar{a} \log \left(\hat{c}+\bar{a} \sqrt{4+\frac{\hat{c}^{2}}{\bar{a}^{2}}}\right)-\bar{a} \log (2 \bar{a}) .
$$

Because $\xi$ is the known spatial coordinate, $\hat{c}(\xi)$ can be obtained from Eq. (144) using a nonlinear root solver and then $\hat{a}(\xi)$ can be obtained from Eq. (142). Moreover, it follows from Fig. 3 and Eqs. (142) and (143) that

$$
\cos \hat{\alpha}=\frac{d \hat{c}}{d \xi}=\frac{2 \bar{a}}{\sqrt{4 \bar{a}^{2}+\hat{c}^{2}}}, \quad \sin \hat{\alpha}=\frac{d \hat{a}}{d \xi}=\frac{\hat{c}}{\sqrt{4 \bar{a}^{2}+\hat{c}^{2}}}, \quad \frac{d \hat{\alpha}}{d \xi}=\frac{4 \bar{a}^{2}}{\left(4 \bar{a}^{2}+\hat{c}^{2}\right)^{3 / 2}} .
$$

Figure 10(a) shows the deformed (solid line) and undeformed (broken line) shapes of an axisymmetric membrane having a parabolic deformed shape and a 


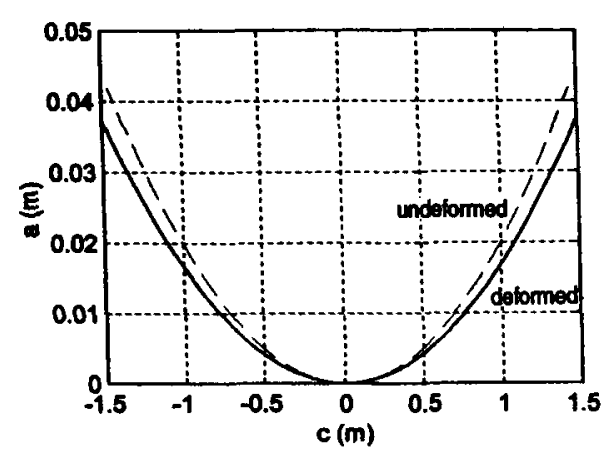

(a)

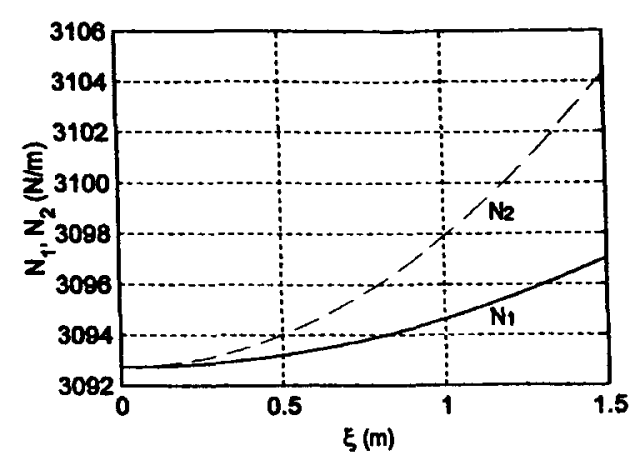

(b)

Fig. 10. The inverse analysis of a known deformed parabolic membrane with $L=1.5 \mathrm{~m}, \bar{a}=10 L$, and $p=200 \mathrm{~Pa}$ : (a) deformed (-) and inverse undeformed (- - ) geometries, and (b) distributions of $N_{1}$ and $N_{2}$.

focus length $\bar{a}=15 \mathrm{~m}, 0 \leq \xi \leq \hat{L}(=1.5 \mathrm{~m})$, and being subjected to an internal pressure of $200 \mathrm{~Pa}$. Figure 10(b) shows the distributions of the internal force intensities $N_{1}$ (along $\xi$ ) and $N_{2}$ (along $\eta$ ). We note that $N_{2}>N_{1}$ and they become equal at $x=0$. Substituting Eq. (145) into Eq. (124) yields

$$
\hat{N}_{1}=\frac{p}{2} \sqrt{4 \bar{a}^{2}+\hat{c}^{2}}, \quad \hat{N}_{2}=\frac{p}{2}\left(\sqrt{4 \bar{a}^{2}+\hat{c}^{2}}+\frac{\hat{c}^{2}}{\sqrt{4 \bar{a}^{2}+\hat{c}^{2}}}\right) .
$$

It is apparent that $\hat{N}_{1}<\hat{N}_{2}$ except that $\hat{N}_{1}=\hat{N}_{2}$ at $\hat{c}=0$ (i.e., the apex). However, Fig. 10(a) shows that the rim diameter at $\xi=L$ needs to be increased in order to have the deformed geometry is parabolic, but it results in $N_{2}>N_{1}$. On the other hand, Fig. 6(b) shows that, if the rim diameter at $x=L$ is fixed, it results in $N_{2}<N_{1}$ but the deformed geometry is nonparabolic. Hence, one can reduce the increase of the rim diameter at $\xi=L$ in Fig. $10(\mathrm{a})$ to make $N_{2}$ close to $N_{1}$ and keep them almost constant at every location. Of course, the deformed geometry will deviate a little from being a paraboloidal surface.

\subsubsection{Cylindrical membrane}

Figure 11(a) shows a known deformed circular cylindrical tube with two spherical ends subjected to an internal pressure $p=2000 \mathrm{~Pa}$. If $L_{1}=2.5 \mathrm{~m}$ and $R_{1}=R_{2}=2 \mathrm{~m}$, Figs. 11(b) and (11c) show the obtained undeformed geometry and the distributions of $e_{1}$ and $e_{2}$, respectively. The undeformed geometry has a sudden change at the junction of the circular tube and the spherical end because the spherical end has a smaller $e_{2}$. If the deformed geometry and $p$ are fixed, $\hat{N}_{1}$ and $\hat{N}_{2}$ are also fixed. Hence, increase of $e_{2}$ requires decrease of $\tilde{Q}_{i j}$, as shown in Eq. (125). In other words, the thickness $h$ of the spherical ends needs to be reduced because $\tilde{Q}_{11}, \tilde{Q}_{22}$, and $\tilde{Q}_{12}$ are proportional to $h$ as shown in Eq. (56). From the geometry shown in Fig. 11(a) and Eq. (124) one can show that $\partial \hat{\alpha} / \partial \xi=0$, 


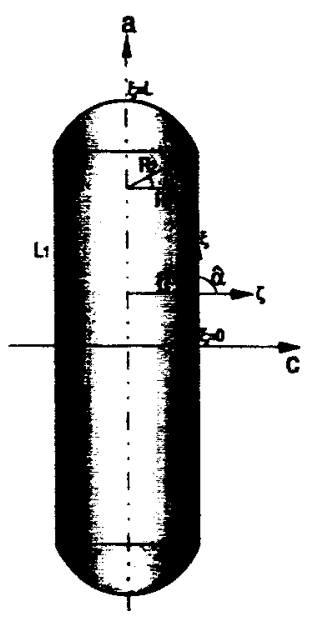

(a)

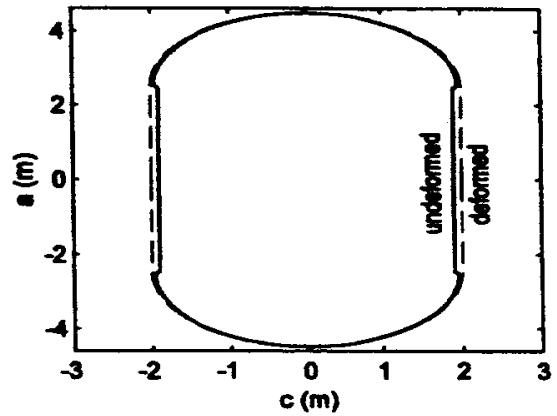

(b)

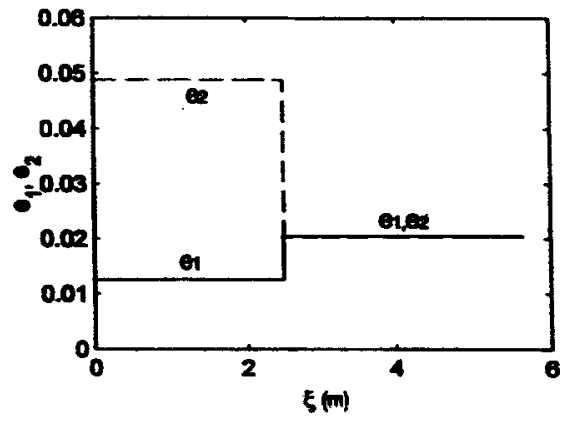

(c)

Fig. 11. The inverse analysis of a known deformed circular cylindrical tube with two spherical ends, $L_{1}=2.5 \mathrm{~m}, R_{1}=R_{2}=2 \mathrm{~m}$, and $p=2000 \mathrm{~Pa}$ : (a) the circular cylindrical membrane, (b) deformed and undeformed geometries, and (c) distributions of $e_{1}$ and $e_{2}$.

$\hat{c} / \sin \hat{\alpha}=R_{1}$, and $\hat{N}_{2}=2 \hat{N}_{1}=p R_{1}$ for the cylindrical tube; and $\partial \hat{\alpha} / \partial \xi=1 / R_{2}$, $\hat{c} / \sin \hat{\alpha}=R_{2}$, and $\hat{N}_{2}=\hat{N}_{1}=p R_{2} / 2$ for the spherical ends. Replacing $\hat{N}_{2}$ and $\hat{N}_{1}$ with $p R_{2} / 2, \tilde{Q}_{i j}$ with $q \tilde{Q}_{i j}$, and $e_{2}$ with the circumferential strain $e_{2 c}$ of the cylindrical tube in Eq. (126) yields

$$
q^{2}\left(\tilde{Q}_{11} \tilde{Q}_{22}-\tilde{Q}_{12}^{2}\right) e_{2 c}+\frac{q\left(\tilde{Q}_{12}-\tilde{Q}_{11}+2 \tilde{Q}_{12} e_{2 c}\right) p R_{2}}{2}-\frac{\left(1+e_{2 c}\right) p^{2} R_{2}^{2}}{4}=0
$$

which can be solved for the $q$ ( $\left.=h_{\text {new }} / h_{\text {original }}\right)$ required to match $e_{2}$ at the junction. Figure 12(a) shows that, if the thickness of the spherical ends is reduced to be $h_{\text {new }}=0.005451 \mathrm{~mm}$ (i.e., $q=0.4292$ from Eq. (147)), the undeformed geometry is smooth at the junction.

Replacing $\hat{N}_{2}$ and $\hat{N}_{1}$ with $p R_{2} / 2$ and $e_{2}$ with the circumferential strain $e_{2 c}$ of the cylindrical tube in Eq. (126) yields

$$
\frac{R_{2}^{2}\left(1+e_{2 c}\right) p^{2}}{4}+\frac{R_{2}\left(\tilde{Q}_{11}-\tilde{Q}_{12}-2 \tilde{Q}_{12} e_{2 c}\right) p}{2}+\left(\tilde{Q}_{12}^{2}-\tilde{Q}_{11} \tilde{Q}_{22}\right) e_{2 c}=0
$$




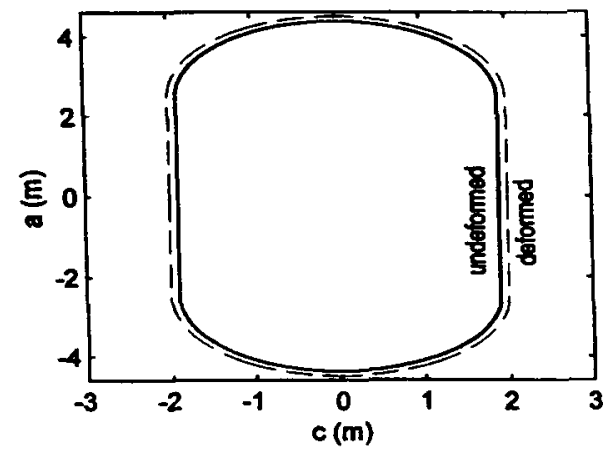

(a)

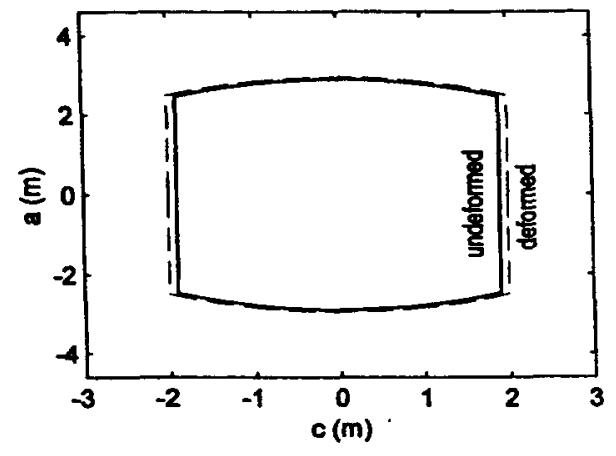

(b)

Fig. 12. The inverse analysis of a known deformed circular cylindrical tube with two spherical ends, $L_{1}=2.5 \mathrm{~m}, R_{1}=2 \mathrm{~m}$, and $p=2000 \mathrm{~Pa}$ : (a) deformed and undeformed geometries if $R_{2}=2 \mathrm{~m}$ and the thickness of the spherical ends is reduced to $0.005451 \mathrm{~mm}$, and (b) deformed and undeformed geometries if the radius of the spherical ends is increased to $R_{2}=4.6597 \mathrm{~m}$.

which can be solved for the $R_{2}$ required to match $e_{2}$ at the junction without changing the thickness of the spherical ends. Figure 12(b) shows that, if $R_{2}$ is increased to $4.6597 \mathrm{~m}$, the undeformed geometry does not have a sudden change at the junction.

\section{Concluding Remarks}

A fully nonlinear membrane theory is derived by treating membranes as shells and using Jaumann strains and stresses and new concepts of local relative displacements and orthogonal virtual rotations. Fully nonlinear governing equations for different membranes are listed, especially the ones for axisymmetric membranes subjected to axisymmetric loading. The membrane theory is used to obtain numerically exact deformed shapes of several axisymmetric membranes subjected to internal pressures. Numerical results show also that bending stiffness is negligible for thin membranes, and natural frequencies of pressurized membranes increase with the internal pressure. Moreover, an inverse solution method for computing the undeformed geometry of a membrane with a known/designed deformed geometry and a known inflation pressure is presented and numerical results are obtained and verified.

\section{Acknowledgments}

This work is supported by the National Science Foundation under Grant CMS9912482 and the NASA Langley Research Center under Grant NAG-1-01037. The support is gratefully acknowledged.

\section{References}

1. M. Salama, M. Lou, and H. Fang, Proceedings of the AIAA Structures, Structural Dynamics and Materials Conference, AIAA-2000-1730, 2000. 
2. M. S. Smith, R. S. Schallenkamp, and L. G. Seely, AIAA International Balloon Technology Conference, San Francisco, CA, June 3-5, 1997.

3. D. Gorinevsky, T. Hyde, and C. Cabuz, Proceedings of the AIAA Structures, Structural Dynamics and Materials Conference, AIAA-2001-1197, 2001.

4. V. Firt, Statics, Formfinding and Dynamics of Air-Supported Membrane Structures, Martinus Nijhoff Publishers, Boston, 1983.

5. A. L. Palisoc, Inflatable Reflector Development Program, Task 3 Report, L'Garde Technical Report, LTR-94-AP-008, 1994.

6. S. V. Damle, M. N. Joshi, S. Sreenivasan, and J. V. Subba Rao, AIAA International Balloon Technology Conference, San Francisco, CA, June 3-5, 1997.

7. P. F. Pai, A. N. Palazotto, and J. M. Greer, Jr., Comput. Struct., 66, 823, 1998.

8. H. Furuya and Y. Kawasaki, Proceedings of the AIAA Structures, Structural Dynamics and Materials Conference, AIAA-2000-1731.

9. G. Greschik, M. M. Mikulas, and A. Palisoc, Proceedings of the AIAA Structures, Structural Dynamics and Materials Conference, AIAA-98-2101, 1998.

10. J. Ruze, Proceedings of the IEEE, 54, 633, 1966.

11. A. Palisoc, G. Veal, C. Cassapakis, G. Greschik, and M. Mikulas, Fifth SPIE Conference on Space Telescopes and Instruments, Kona, Hawaii, March, 1998.

12. P. F. Pai and A. H. Nayfeh, Nonlinear Dynamics, 6, 459, 1994.

13. P. F. Pai and A. N. Palazotto, J. Eng. Mech., 121, 568, 1995.

14. P. F. Pai and A. H. Nayfeh, Nonlinear Dynamics, 2, 445, 1991.

15. K. Washizu, Variational Methods in Elasticity \& Plasticity, 3rd edition, Pergamon Press Inc., New York, 1982.

16. P. F. Pai and A. N. Palazotto, Int. J. Solids Struct., 32, 3047, 1995.

17. H. Golden, T. W. Strganac, and R. A. Schapery, AIAA International Balloon Technology Conference, San Francisco, CA, June 3-5, 1997.

18. J. Stoer and R. Bulirsch, Intraduction to Numerical Analysis, Springer-Verlag, New York, 1980. 